\title{
R7 photoreceptor axon targeting depends on the relative levels of lost and found expression in $\mathrm{R7}$ and its synaptic partners
}

\author{
Jessica Douthit ${ }^{1,4}$, Ariel Hairston ${ }^{1,4}$, Gina Lee ${ }^{1}$, Carolyn A. Morrison ${ }^{1}$, Isabel Holguera ${ }^{2}$ and \\ Jessica E. Treisman ${ }^{1,3}$
}
1) Kimmel Center for Biology and Medicine at the Skirball Institute and Department of Cell Biology, NYU School of Medicine, 540 First Avenue, New York, NY 10016, USA

2) Department of Biology, New York University, New York, NY 10003, USA

3) Corresponding author: Tel: 212-263-1031, Fax: 212-263-7760, Jessica.Treisman@nyulangone.org

4) These two authors contributed equally. 


\begin{abstract}
As neural circuits form, growing processes select the correct synaptic partners through interactions between cell surface proteins. The presence of such proteins on two neuronal processes may lead to either adhesion or repulsion; however, the consequences of mismatched expression have rarely been explored. Here we show that the Drosophila CUB-LDL protein Lost and found (Loaf) is required in the UV-sensitive R7 photoreceptor for normal axon targeting only when Loaf is also present in its synaptic partners. Although targeting occurs normally in loaf mutant animals, removing loaf from photoreceptors or expressing it in their postsynaptic neurons Tm5a/b or Dm9 in a loaf mutant causes mistargeting of R7 axons. Loaf localizes primarily to intracellular vesicles including endosomes. We propose that Loaf regulates the trafficking or function of one or more cell surface proteins, and an excess of these proteins on the synaptic partners of $\mathrm{R} 7$ prevents the formation of stable connections.
\end{abstract}




\section{Introduction}

During nervous system development, growing axons must navigate through a complex environment and select the correct synaptic partners from numerous potential choices. Recognition of cell surface molecules plays an important role in axon guidance and targeting and the establishment of specific synaptic connections (Yogev and Shen 2014). Interactions between cell surface molecules can lead to either adhesion or repulsion, and their relative levels on different cells are important for appropriate connections to form. For instance, gradients of ephrins and their Eph receptors enable retinal axons to form a topographic map in visual areas of the brain because Eph levels determine the sensitivity to ephrins (Triplett and Feldheim 2012). In the Drosophila olfactory system, olfactory receptor neurons preferentially connect to projection neurons that express matching levels of the adhesion molecule Teneurin (Hong et al. 2012). As defects in synaptic adhesion molecules can lead to autism and other neurodevelopmental disorders (Van Battum et al. 2015; Gilbert and Man 2017), identifying mechanisms that regulate synaptic partner choice is likely to enhance our understanding of such human diseases.

The Drosophila visual system has been a fruitful model for investigations of circuit assembly and synaptic specificity (Plazaola-Sasieta et al. 2017). The two color photoreceptors in the fly retina, R7 and R8, project to distinct layers in the medulla, M6 and M3 respectively. The R7 growth cone first actively targets a temporary layer, and then passively reaches its final layer due to the growth of other neuronal processes (Ting et al. 2005; Ozel et al. 2015). Early stabilization of the R7 and R8 growth cones in different layers depends on differences in their relative levels of the transcription factor Sequoia (Seq); the adhesion molecule N-cadherin (Ncad) is thought to be the relevant target of Seq in these cells (Petrovic and Hummel 2008; Kulkarni et al. 2016). Both Ncad and the receptor protein tyrosine phosphatase (RPTP) Lar are required to stabilize $\mathrm{R} 7$ terminals in the M6 layer. In the absence of either protein they remain in the M3 layer, although defects are observed earlier in development in Ncad mutants than in Lar mutants (Clandinin et al. 2001; Lee et al. 2001; Maurel-Zaffran et al. 2001; Ting et al. 2005; Ozel et al. 2015; Ozel et al. 2019). Another RPTP, Ptp69D, is partially redundant with Lar, and the depth of R7 axon termination correlates with the total level of RPTP activity (Newsome et 
al. 2000; Hofmeyer and Treisman 2009; Hakeda-Suzuki et al. 2017). Stabilization of R7 contacts also requires the presynaptic proteins Liprin- $\alpha$ and Syd-1 that act downstream of Lar (Choe et al. 2006; Hofmeyer et al. 2006; Holbrook et al. 2012; Ozel et al. 2019).

The primary synaptic targets of $\mathrm{R} 7$ that are responsible for its function in driving the spectral preference for ultraviolet light are the Dm8 medulla interneurons (Gao et al. 2008; Takemura et al. 2013; Karuppudurai et al. 2014; Ting et al. 2014). These cells fall into two subclasses, yellow ( $y$ ) and pale (p), and their survival depends on their correct pairing with the appropriate R7 cell subtype, expressing either Rh4 (yR7) or Rh3 (pR7) (Courgeon and Desplan 2019; Menon et al. 2019). The synapses R7 cells form on Dm8 cells often include the projection neurons Tm5a (for yR7s) or Tm5b (for pR7s) as a second postsynaptic element (Gao et al. 2008; Takemura et al. 2013; Menon et al. 2019). Another interneuron, Dm9, is both pre- and postsynaptic to R7 and R8 and mediates inhibitory interactions between ommatidia (Takemura et al. 2013; Takemura et al. 2015; Heath et al. 2020). It is not known which, if any, of these cell types provide Ncad or RPTP ligands that stabilize filopodia from the R7 growth cone (Yonekura et al. 2007; Hofmeyer and Treisman 2009; Hakeda-Suzuki et al. 2017; Ozel et al. 2019). Glia are also involved in establishing the pattern of R7 synaptogenesis, as they prevent excessive synapse formation through the adhesion protein Klingon $(\mathrm{Klg})$ and its partner CDIP (Shimozono et al. 2019).

Here we identify a novel CUB-LDL domain transmembrane protein, Lost and found (Loaf), that acts in photoreceptors to promote the formation of stable R7 contacts in the M6 layer. R7 mistargeting to the M3 layer is observed when loaf function is lost from photoreceptors, but not in a fully loaf mutant animal. Similar defects can be induced in loaf mutants by expressing Loaf in neurons that include Tm5a, Tm5b, Dm9 and Dm8, suggesting that $\mathrm{R} 7$ targeting is disrupted when Loaf is absent from R7 but present in its postsynaptic partners. Loaf does not itself promote cell adhesion and localizes primarily to endosomes. We propose that Loaf controls the trafficking or function of cell surface molecules that are used to match R7 to the correct postsynaptic neurons. 


\section{Results}

lost and found is required in photoreceptors for normal R7 axon targeting

A microarray-based screen for genes with enriched expression in the R7 and R8 photoreceptors relative to R1-R6 identified CG6024, which encodes an uncharacterized transmembrane protein (Pappu et al. 2011). CG6024 is also a predicted target of Glass (NavalSanchez et al. 2013), a transcription factor required for photoreceptor differentiation and axon guidance (Moses et al. 1989; Selleck and Steller 1991). To test whether CG6024 has a function in axon targeting by R7 or R8, we expressed RNAi transgenes targeting CG6024 with two different drivers: GMR-GAL4 drives expression in all differentiating cell types in the eye (Freeman 1996), and removing a stop cassette from Actin>CD2>GAL4 with the eye-specific recombinase ey ${ }^{3.5}$-FLP (Bazigou et al. 2007) leads to RNAi expression in the entire eye disc. In both cases, R8 targeting was unaffected, but we observed a loss of R7 terminals from the M6 layer of the medulla (Figure $1 \mathrm{~A}-\mathrm{C}$ ); $30-60 \%$ of $\mathrm{R} 7$ axons were mistargeted to the $\mathrm{M} 3$ layer (Figure 1D-F). This phenotype appears to arise during the second stage of R7 targeting, when filopodia are stabilized to form synapses (Ting et al. 2005; Ozel et al. 2019). R7 axons targeted correctly to their temporary layer at $40 \mathrm{~h}$ after puparium formation (APF) when CG6024 was knocked down, but many terminals did not reach or were not stabilized in their permanent target layer, M6, at $60 \mathrm{~h}$ APF (Figure 1G-K). We named the gene lost and found (loaf) based on the failure of R7 axons lacking loaf to find the right target layer and on the rescue of this phenotype discussed below. The Loaf protein contains extracellular CUB and LDLa domains and a predicted transmembrane domain (Figure $2 \mathrm{~A}$ ), making it a candidate to directly mediate target recognition by $\mathrm{R} 7$.

\section{loaf mutant R7 axons show targeting defects only when loaf is present in other cells}

In the experiments above, we used two independently generated RNAi lines targeting the same region of the gene to knock down loaf (Figure 2A), both of which produced similar R7 mistargeting phenotypes (Figure 1D) . To confirm that this phenotype was due to loss of loaf rather than an off-target effect of the RNAi, we used the CRISPR-Cas9 system to generate 

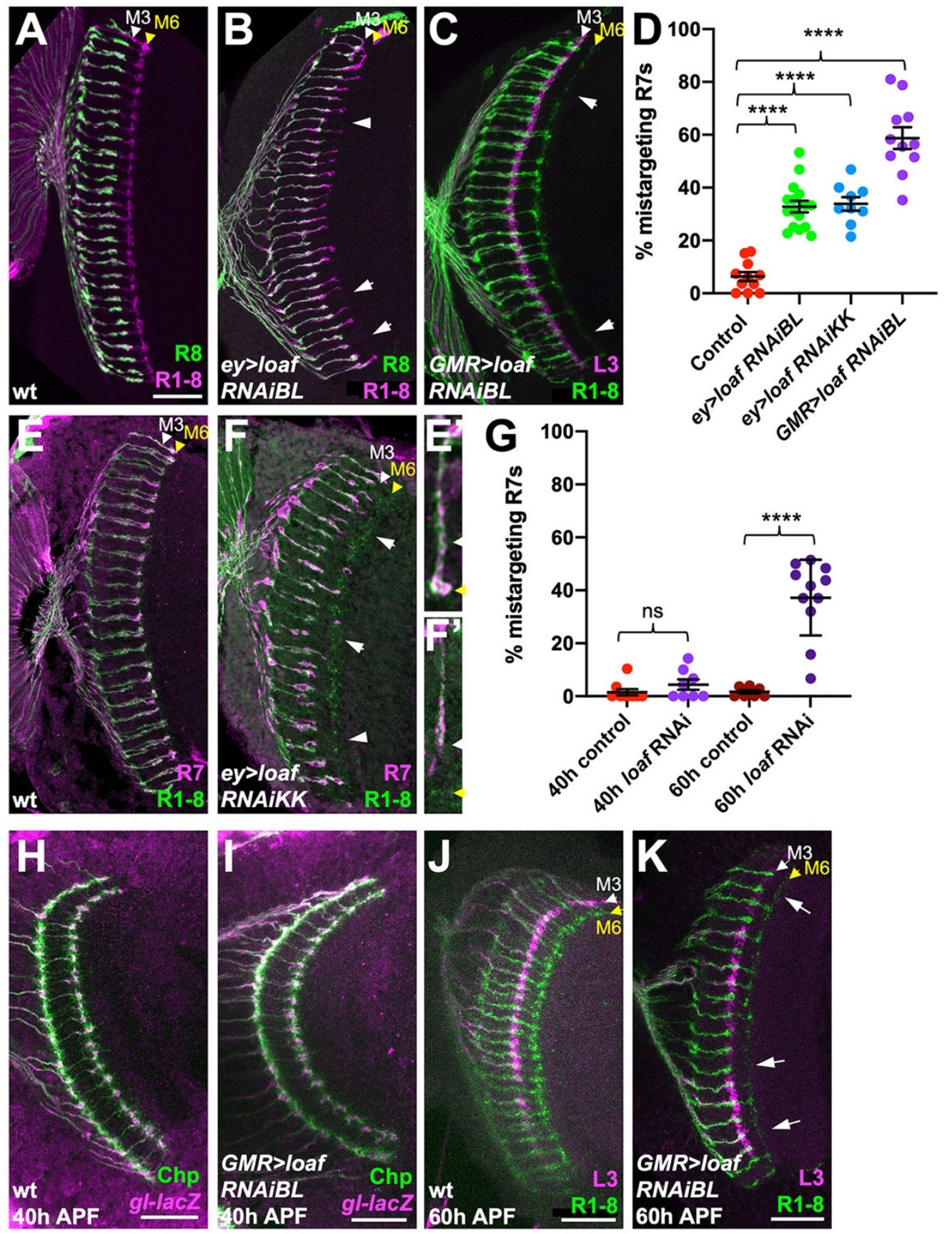

Douthit et al. Figure 1 
Figure 1: loaf RNAi in photoreceptors causes $\mathbf{R 7}$ mistargeting. ( $A-C, E, F)$ cryostat sections of adult heads stained for Chaoptin (Chp) to label all photoreceptor axons (magenta in A, B, green in C, E, F), Rh5-GFP and Rh6-GFP to label R8 (green in A, B), 22E09-LexA driving LexAop-myrtdTomato to label L3, which projects to the M3 layer (magenta in C) or panR7-lacZ to label R7 (magenta in E, F). (A, E) wild type; (B) ey3.5-FLP, Act>CD2>GAL4; UAS-dcr2; UAS-loaf RNAiBL (P\{TRiP.JF03040\}attP2); (C) GMR-GAL4, UAS-dcr2; UAS-loaf RNAiBL; (F) ey3.5-FLP, Act>CD2>GAL4; UAS-dcr2; UAS-loaf RNAiKK (P\{KK112220\}VIE-260B). Arrows show examples of R7 mistargeting. White arrowheads indicate the M3 layer and yellow arrowheads the M6 layer. $\left(E^{\prime}, F^{\prime}\right)$ show enlargements of single $R 7$ axons from (E, F). (D) quantification of the percentage of $\mathrm{R} 7$ axons that failed to reach the $\mathrm{M} 6$ layer in the same genotypes. $\mathrm{n}=11$ (control, $G M R>R N A i B L$ ), $16($ ey $>R N A i B L)$, or $9(e y>R N A i K K) . * * * *, p<0.0001$ by unpaired t-test. Error bars show mean \pm standard error of the mean (SEM) in this and all other graphs. (H-K) pupal brains stained for Chp (green) and glass ( $g l$ )-lacZ, which labels all photoreceptor axons (magenta in $\mathrm{H}, \mathrm{I}$ ) or 22E09-LexA driving LexAop-myr-tdTomato to label L3 neuronal processes in the M3 layer (magenta in J, K). $(\mathrm{H}, \mathrm{I}) 40 \mathrm{~h}$ after puparium formation (APF); (J, K) $60 \mathrm{~h}$ APF. (H, J) wild type (GMR-GAL4, UASdcr2/+); (I, K) GMR-GAL4, UAS-dcr2; UAS-loaf RNAiBL. Loss of loaf does not prevent the initial targeting of R7 axons to their temporary layer at $40 \mathrm{~h} \mathrm{APF,} \mathrm{but} \mathrm{many} \mathrm{axons} \mathrm{fail} \mathrm{to} \mathrm{project}$ beyond that layer at $60 \mathrm{~h} \mathrm{APF.} \mathrm{(G)} \mathrm{quantification} \mathrm{of} \mathrm{the} \mathrm{percentage} \mathrm{of} \mathrm{R7} \mathrm{axons} \mathrm{that} \mathrm{did} \mathrm{not}$ reach the appropriate layer for these genotypes and stages. $\mathrm{n}=9$ (40 h control), 8 (40 h RNAi, 60 h control) or 11 (60 h RNAi). ${ }^{* * * *}, \mathrm{p}<0.0001$ by unpaired t-test with Welch's correction; ns, not significant. Scale bars, $20 \mu \mathrm{m}$. 
deletion alleles that removed the LDLa, transmembrane and cytoplasmic domains of the protein (Figure $2 \mathrm{~A}, \mathrm{I}$ ). The sgRNAs were directed against a region of the gene distinct from the RNAi target sequence, and using them to delete the loaf gene in the eye by somatic CRISPR reproduced the $\mathrm{R} 7$ targeting defect (Figure 2A, B, H). Surprisingly, germline removal of loaf resulted in homozygous mutant flies that were viable and showed largely normal R7 targeting (Figure 2C, D, H), indicating that global loss of loaf does not affect this process. Expressing loaf RNAi had no effect in this loaf mutant background (Figure $2 \mathrm{E}, \mathrm{H}$ ), confirming that the RNAi phenotype was due to its effect on loaf rather than another gene. Together, these results indicate that the phenotype caused by removing loaf from the eye is dependent on the presence of loaf in the optic lobes. R7 targeting may therefore depend on the amount of Loaf in $\mathrm{R} 7$ relative to other cells rather than its absolute presence or absence.

To test this hypothesis, we generated clones of cells in the eye that were homozygous for loaf deletion alleles in an otherwise heterozygous background. As predicted, these showed mistargeting of R7 axons to the M3 layer (Figure $2 \mathrm{~F}, \mathrm{H}$, Figure 2 - figure supplement $1 \mathrm{~A}$ ). The mistargeting was significantly rescued by expressing either HA-tagged or untagged Loaf within the mutant clones (Figure 2G, H, Figure 2 - figure supplement 1B-D), confirming that it is due to loss of loaf from photoreceptors. These results could be explained if correct targeting depends on the relative levels of Loaf in R7 and another cell type. Loss of Loaf in R7 when it is present in the other cell type would cause mistargeting. When Loaf is absent from all cells, redundant mechanisms may be sufficient to maintain R7 terminals in the correct layer.

\section{Loaf levels in Dm8, the major synaptic target of R7, do not affect R7 targeting}

The medulla interneuron Dm8, which mediates the preference for ultraviolet over visible light, was reported to be the major postsynaptic target of R7 (Gao et al. 2008; Takemura et al. 2013; Ting et al. 2014). We therefore considered the hypothesis that R7 and its postsynaptic partner Dm8 must both express Loaf to form a stable connection (Figure 3F). We first determined the effect of removing loaf function from Dm8. Expressing loaf RNAi or Cas9 and loaf sgRNAs in neurons that include Dm8 cells with DIP- $\gamma$-GAL4 or traffic jam (tj)-GAL4 (Carrillo et al. 2015; Courgeon and Desplan 2019) did not cause any R7 targeting phenotype 

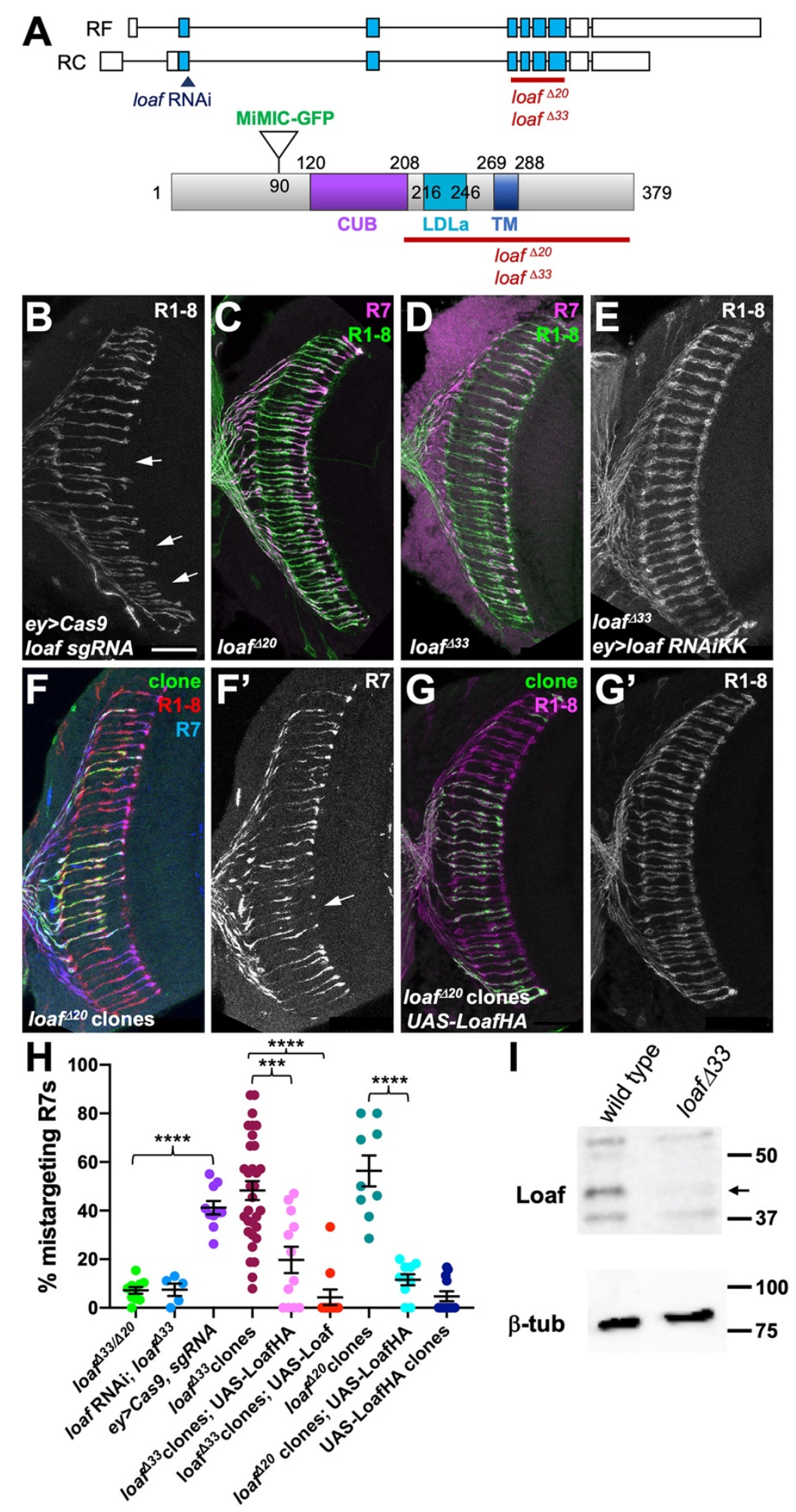

\section{Douthit et al. Figure 2}


Figure 2: R7 is only affected by eye-specific loss of loaf. (A) diagrams of the loaf gene and protein. Coding exons, which are identical for the two isoforms, are shown as blue boxes and non-coding exons as white boxes. The region targeted by both RNAi lines, the MiMIC GFP insertion and the extent of the loaf $f^{\Delta 20}$ and loaf $f^{\Delta 33}$ deletions are indicated. These two deletions were independently generated and have minor sequence differences around the cut site. TM, transmembrane domain. (B-G) cryostat sections of adult heads stained for Chp (B, E, G', green in $C, D$, red in F, magenta in $G$ ), panR7-lacZ ( $F^{\prime}$, magenta in $C, D$, blue in F), and GFP (green in F, G). (B) ey3.5-FLP, Act>CD2>GAL4; loaf sgRNAs; UAS-Cas9P2; (C) loaf ${ }^{\Delta 20}$ homozygote; (D) loaf 433 homozygote; (E) ey3.5-FLP, Act>CD2>GAL4; UAS-dcr2/UAS-loaf RNAiKK; loaf ${ }^{\triangle 33}$; (F) loaf ${ }^{\Delta 20}$ clones positively labeled with IGMR-GAL4, UAS-GFP; (G) loaf ${ }^{420}$ clones expressing UAS-LoafHA with IGMR-GAL4, positively labeled with GFP. Scale bar, $20 \mu \mathrm{m}$. (H) quantification of the percentage of $\mathrm{R} 7$ axons that failed to reach the $M 6$ layer in the indicated genotypes. $n=10$ (loaf ${ }^{\Delta 33} /$ loaf $^{\Delta 20}$; ey>Cas9, sgRNA; loaf ${ }^{\Delta 20}$ clones, UAS-LoafHA), 5 (loafRNAi; loaf ${ }^{\Delta 33}$ ), 32 (loaf $^{\Delta 33}$ clones), 12 (loaf $\triangle 33$ clones, UAS-LoafHA; wild type clones, UAS-LoafHA), 11 (loaf ${ }^{433}$ clones, UASLoaf) or 9 (loaf ${ }^{\Delta 20}$ clones). Error bars show mean \pm SEM. $* * *, p<0.0005 ; * * * *, p<0.0001$ by unpaired t-test, with Welch's correction when variances are significantly different. loaf homozygotes show little R7 mistargeting, but are resistant to the effect of loaf RNAi. R7 mistargeting is observed when loaf sgRNAs and Cas9 are expressed in the eye, and in clones homozygous for loaf alleles. This clonal phenotype is rescued by expressing UAS-LoafHA or UASLoaf in the mutant cells. (I) Western blot of extracts from wild type and loaf $f^{\triangle 33}$ larval brains using an antibody to the cytoplasmic domain of Loaf and $\beta$-tubulin antibody as a loading control. Loaf protein (arrow) is absent in loaf 433 mutants. 

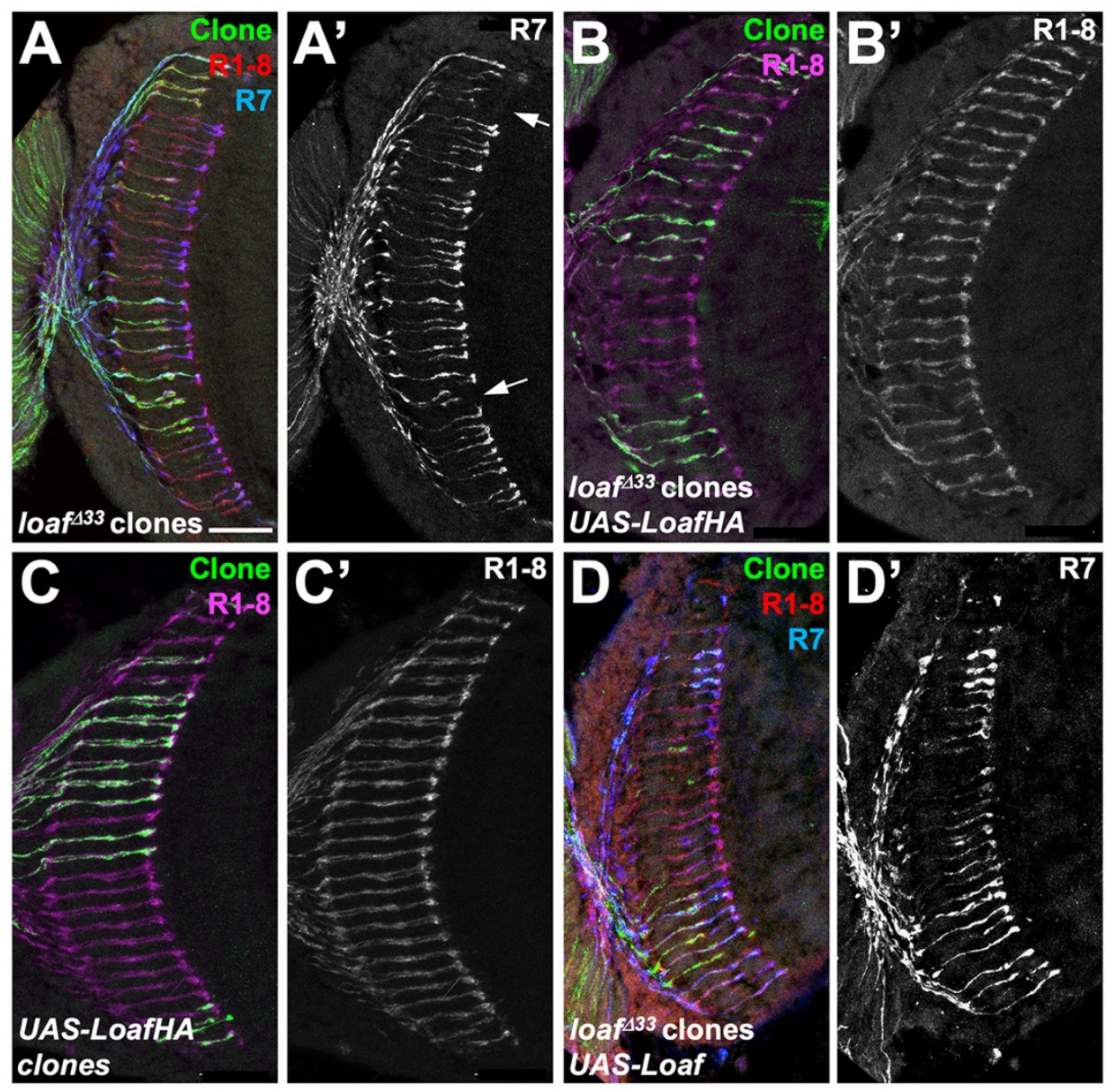

Figure 2 - figure supplement 1: R7 mistargeting in loaf mutant clones is rescued by tagged or untagged Loaf. Cryostat sections of adult heads with clones positively labeled with GFP, stained for Chp ( $B^{\prime}, C^{\prime}$, red in $A, D$, magenta in $\left.B, C\right)$, GFP (green) and panR7-lacZ ( $A^{\prime}, D^{\prime}$, blue in $\left.A, D\right)$. (A) loaf ${ }^{\Delta 33}$ clones; (B) loaf ${ }^{\Delta 33}$ clones expressing UAS-LoafHA with IGMR-GAL4; (C) clones expressing UAS-LoafHA with IGMR-GAL4; (D) loaf ${ }^{133}$ clones expressing UAS-Loaf with IGMR-GAL4. Scale bar, $20 \mu \mathrm{m}$. 
(Figure 3 - figure supplement 1A, B). As it was difficult to assess the reduction in Loaf levels caused by these manipulations, we generated loaf mutant clones in the brain and labeled the mutant Dm8 cells with ortc2b-GAL4 (Ting et al. 2014). R7 axons that contacted the dendrites of mutant Dm8 cells correctly reached the M6 layer, and there was no obvious defect in the position or morphology of the mutant Dm8 dendrites (Figure 3A, B).

We predicted that expressing Loaf in Dm8 cells in a loaf mutant background would result in a mismatch between $\mathrm{R} 7$ and $\mathrm{Dm} 8$ that would be similar to removing loaf from $\mathrm{R} 7$ in a wild-type background (Figure 3F). We tested this by expressing UAS-LoafHA in loaf mutant flies with the Dm8 drivers DIP- $\gamma$-GAL4, tj-GAL4 and drifter (drf)-GAL4 (Hasegawa et al. 2011; Carrillo et al. 2015; Courgeon and Desplan 2019), as well as a combination of tj-GAL4 and DIP- $\gamma$-GAL4. However, we did not observe significant levels of R7 mistargeting (Figure 3C-E, Figure 3 - figure supplement $1 C, D)$, arguing against a requirement for matching Loaf levels in $\mathrm{R} 7$ and Dm8.

\section{Loaf levels in cholinergic and glutamatergic neurons influence R7 targeting}

Since the presence or absence of Loaf in Dm8 did not appear to affect R7 targeting, we searched for other Loaf-expressing cells that might interact with R7. We used several methods to examine the location of Loaf expression in the brain. RNA-Seq analysis of sorted cell types in the adult brain revealed widespread expression of loaf, although at varying levels (Konstantinides et al. 2018; Davis et al. 2020). However, Loaf translation in photoreceptors reaches its maximum at mid-pupal stages, when $\mathrm{R} 7$ axons are targeting the M6 layer (Ting et al. 2005; Zhang et al. 2016), so adult expression levels in other cells might not be reflective of this developmental stage. At pupal stages, we observed that a GFP protein trap insertion in loaf was expressed in many cells in the medulla (Figure 2A, Figure 4 - figure supplement 1A). Finally, we generated an antibody that recognizes the cytoplasmic domain of Loaf (Figure 2I). As this antibody cross-reacted with another protein present in the cell bodies of medulla neurons (Figure 4C), we could only evaluate Loaf expression within the neuropil. In pupal brains, Loaf was enriched in specific layers of the medulla neuropil and also in R7 axons and terminals (Figure 4A; Figure 4 - figure supplement 1C). This staining was absent in loaf mutants (Figure 4C), and the enrichment in R7 processes was specifically lost when loaf RNAi was expressed 

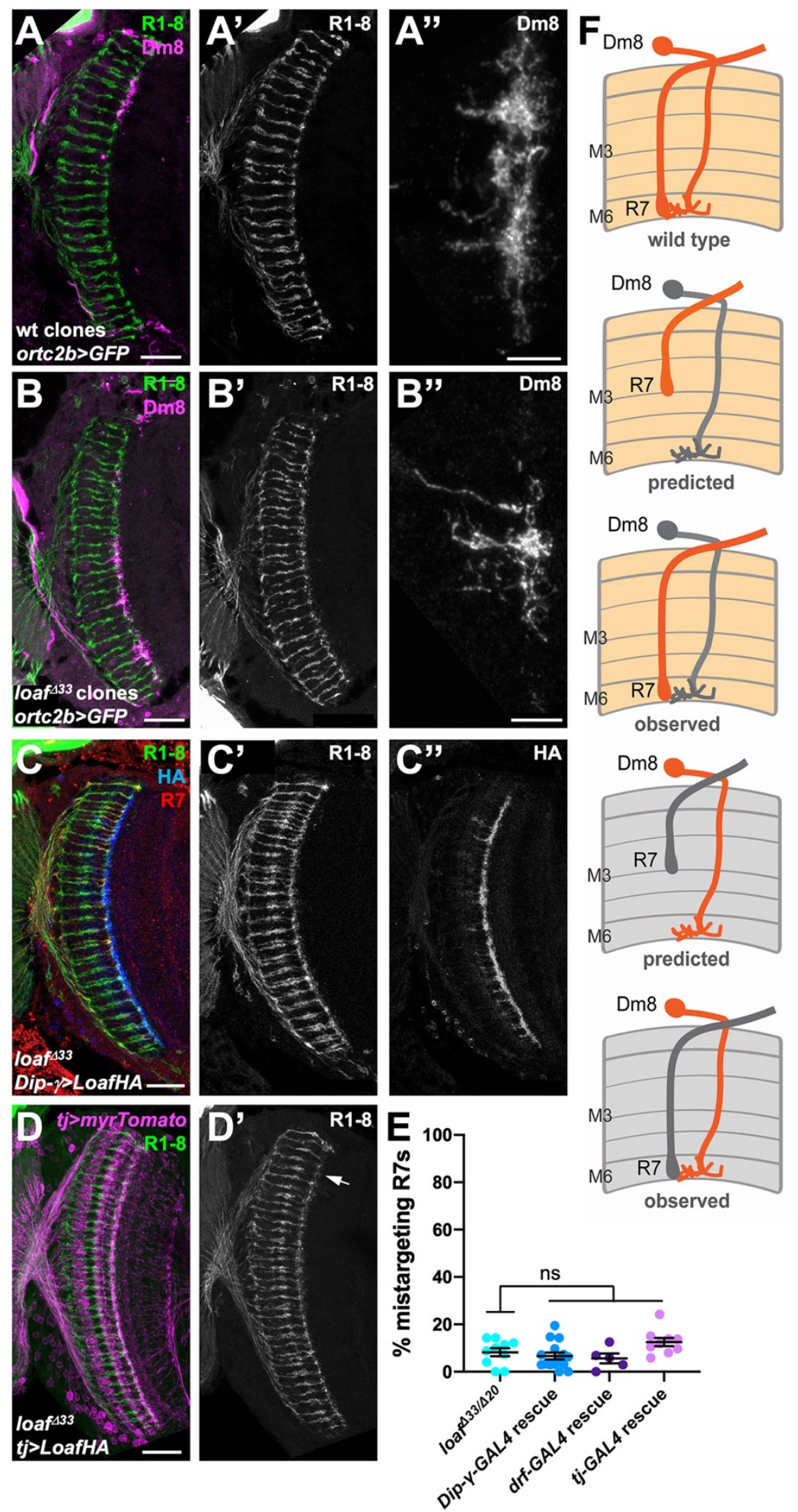

Douthit et al. Figure 3 
Figure 3: Changing the level of Loaf in Dm8 does not affect R7 targeting. (A-D) cryostat sections of adult heads stained for Chp ( $A^{\prime}-D^{\prime}$, green in $\left.A-D\right)$, GFP ( $A^{\prime \prime}, B^{\prime \prime}$, magenta in $\left.A, B\right)$, panR7-lacZ (red in C), HA ( $C^{\prime \prime}$, blue in C) or myrTomato (magenta in $\left.\mathrm{D}\right)$. (A) wild type clones in which Dm8 is labeled with ortc2b-GAL4, UAS-CD8GFP; (B) loaf ${ }^{433}$ clones in which Dm8 is labeled with ortc2b-GAL4, UAS-CD8GFP. (A" and B" show enlargements of labeled Dm8 dendrites. loaf mutant Dm8 dendrites and the R7 axons that target them have the normal position and

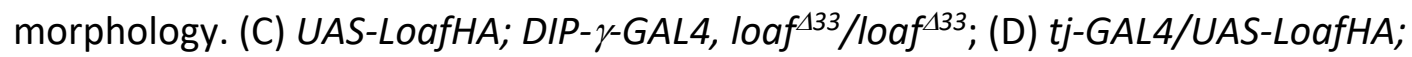
loaf $f^{\Delta 33} /$ loaf $f^{\Delta 33}$. The arrow in ( $\left.D^{\prime}\right)$ indicates minor R7 mistargeting that was not statistically significant. Scale bars, $20 \mu \mathrm{m}(A-D), 5 \mu \mathrm{m}\left(\mathrm{A}^{\prime \prime}, \mathrm{B}^{\prime \prime}\right)$. (E) quantification of the percentage of R7 axons that failed to reach the M6 layer in the indicated genotypes. $\mathrm{n}=10\left(\right.$ loaf $f^{\Delta 33} /$ loaf $^{\Delta 20}$; DIP- $\gamma$ GAL4 rescue; $t j-G A L 4$ rescue), or 5 (drf-GAL4 rescue). Error bars show mean \pm SEM. ns, not significant by unpaired t-test. Expressing Loaf in Dm8 neurons in a loaf mutant does not cause R7 mistargeting. (F) diagrams explaining the predicted results if Loaf expression in $\mathrm{R} 7$ has to match its expression in Dm8. R7 and Dm8 both express Loaf (orange), which is also present in other cells in the brain. Removing loaf from Dm8 (gray) or expressing Loaf in Dm8 in a loaf mutant (gray in R7 and brain) would cause a mismatch and is predicted to result in R7 mistargeting. However, (A-E) show that there is no mistargeting in these situations (observed), indicating that Loaf does not act in Dm8 to regulate $\mathrm{R} 7$ targeting. 

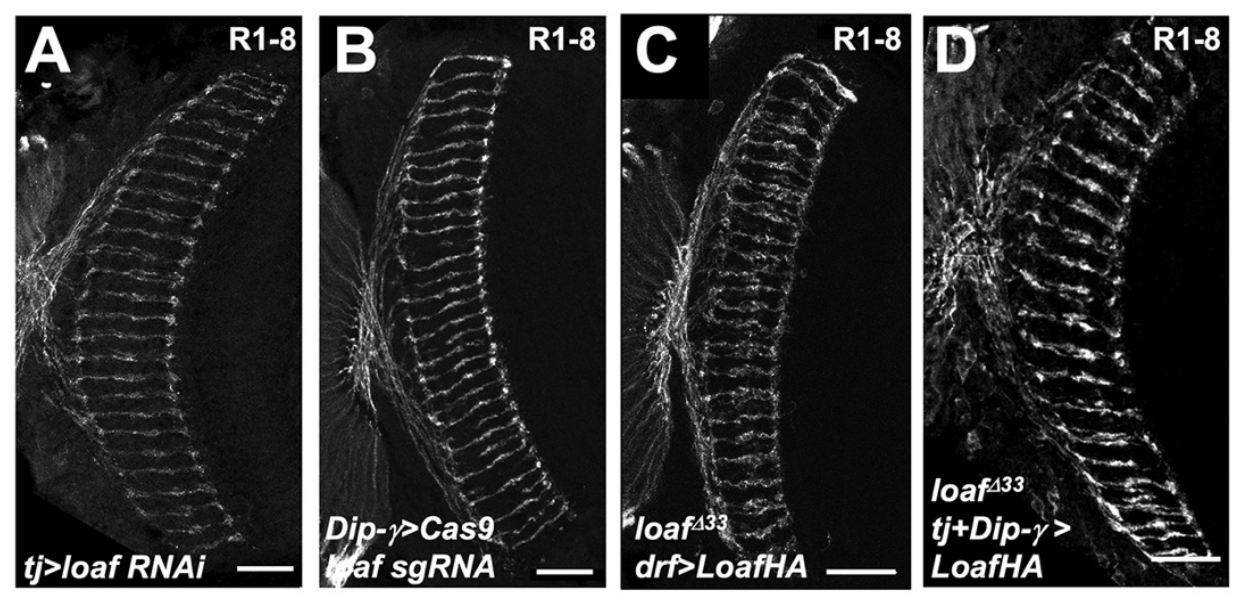

Figure 3 - figure supplement 1: Changing Loaf levels in Dm8 has no effect. Cryostat sections of adult heads stained for Chp. (A) tj-GAL4; loaf RNAiBL; (B) loaf sgRNAs; DIP- $\gamma$-GAL4/UAS-Cas9P2;

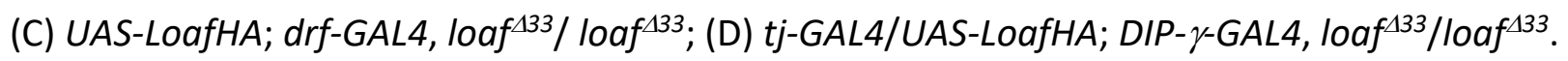
Scale bar, $20 \mu \mathrm{m}$.

with GMR-GAL4 (Figure 4B; Figure 4 - figure supplement 1D). The Loaf protein trap was primarily present in cell bodies and was not visibly enriched in R7 axons; we believe that this insertion disrupts the normal localization of the protein, as clones homozygous for the insertion showed R7 mistargeting (Figure 4 - figure supplement 1A, B). When misexpressed in photoreceptors, LoafHA was efficiently transported to R7 axons and terminals (Figure 4 - figure supplement 1F). Consistent with our findings, recent single-cell RNA-Seq data from dissociated optic lobes show that significant loaf expression is present in almost every cluster throughout the pupal stage, although its levels are generally lower in clusters identified as glia. loaf expression in photoreceptors is highest at P40 and P50, but declines at later stages (Kurmangaliyev et al. 2020; Ozel et al. 2021).

Because these data did not identify a specific cell type that would be most likely to interact with R7 using Loaf, we tested whether R7 mistargeting could be induced by expressing Loaf in broad categories of cells in a loaf mutant background. We observed no phenotype when 

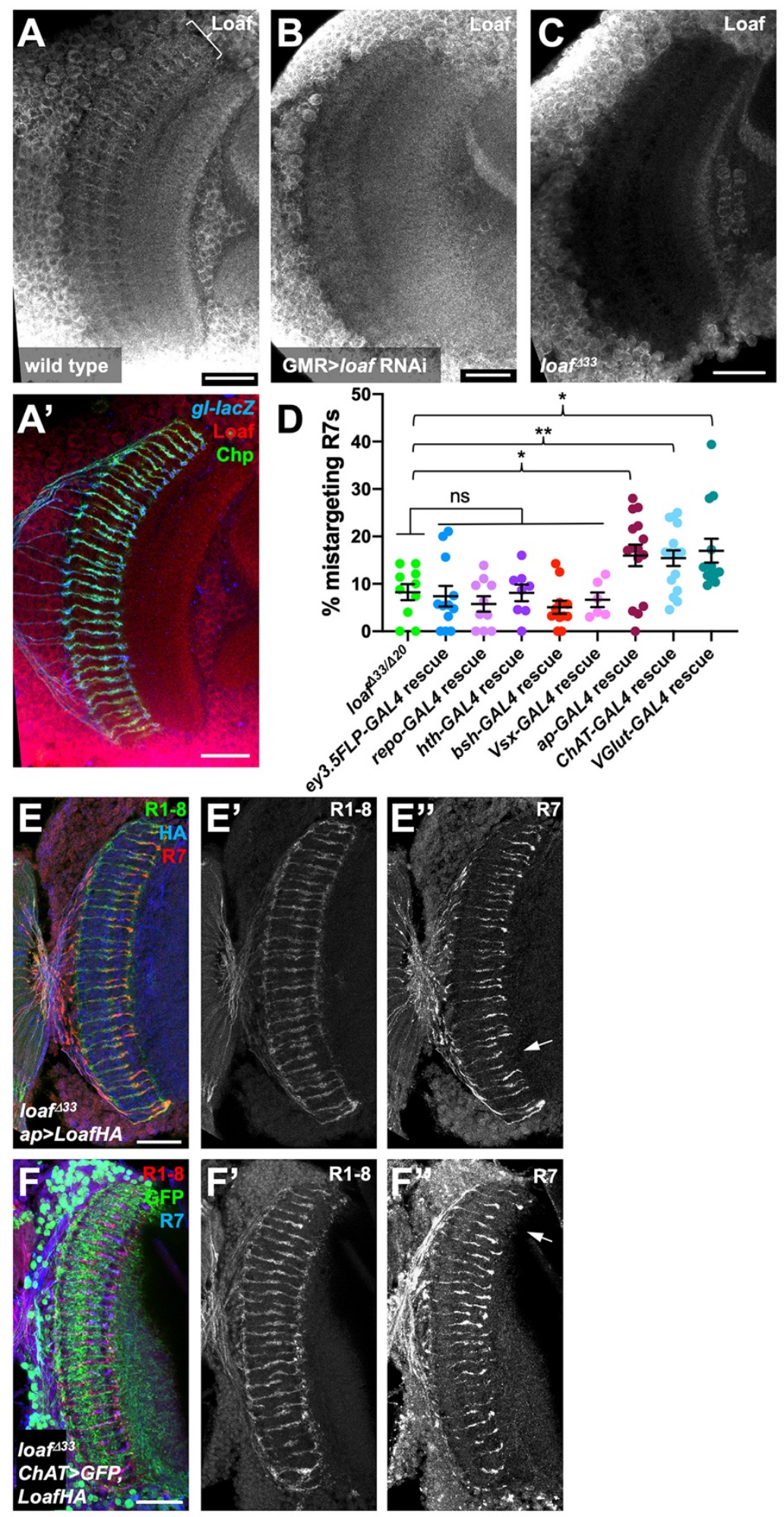

\section{Douthit et al. Figure 4}


Figure 4: Loaf levels in cholinergic and glutamatergic neurons influence R7 targeting. (A-C) pupal brains at $60 \mathrm{~h}$ APF stained for Loaf (A-C, red in $\mathrm{A}^{\prime}$ ), Chp (green in $\mathrm{A}^{\prime}$ ) and gl-lacZ (blue in $\left.\mathrm{A}^{\prime}\right)$. (A) wild type; (B) GMR-GAL4, UAS-dcr2; UAS-loaf RNAiBL; (C) loaf ${ }^{\triangle 33}$. Loaf antibody staining in the medulla neuropil is absent in the loaf mutant (C). Enriched staining in $\mathrm{R} 7$ axons (bracket in $A$ ) is lost when loaf is knocked down in photoreceptors (B). The antibody appears to crossreact with a protein present in medulla cell bodies, as this staining is still present in loaf mutant brains (C). (D) quantification of the percentage of R7 axons that failed to reach the M6 layer in the indicated genotypes. $\mathrm{n}=10$ (loaf $\Delta 33 /$ loaf ${ }^{\Delta 20}$; repo-GAL4 rescue) 12 (ey3.5-FLP, Act>CD2>GAL4 rescue) 8 (hth-GAL4 rescue), 11 (bsh-GAL4 rescue), 6 (VsX-GAL4 rescue), 15 (ap-GAL4 rescue), 16 (ChAT-GAL4 rescue) or 13 (vGlut-GAL4 rescue). Error bars show mean \pm SEM. *, $\mathrm{p}<0.05 ; * *$, $\mathrm{p}<0.01 ; \mathrm{ns}$, not significant by unpaired t-test. Expressing Loaf in cholinergic or glutamatergic neurons or in the precursors of cholinergic neurons with ap-GAL4 in a loaf mutant causes R7 mistargeting. (E, F) cryostat sections of adult heads stained for Chp ( $E^{\prime}, F^{\prime}$, green in $E$, red in $F$ ), panR7-lacZ ( $\mathrm{E}^{\prime \prime}, \mathrm{F}^{\prime \prime}$, red in $\mathrm{E}$, blue in $\mathrm{F}$ ), $\mathrm{HA}$ (blue in $\mathrm{E}$ ) or GFP (green in $\mathrm{F}$ ). (E) ap-GAL4/UASLoafHA; loaf ${ }^{\Delta 33}$; (F) ChAT-GAL4, UAS-CD8GFP/UAS-LoafHA; loaf ${ }^{\Delta 33}$. Scale bars, $20 \mu \mathrm{m}$. 

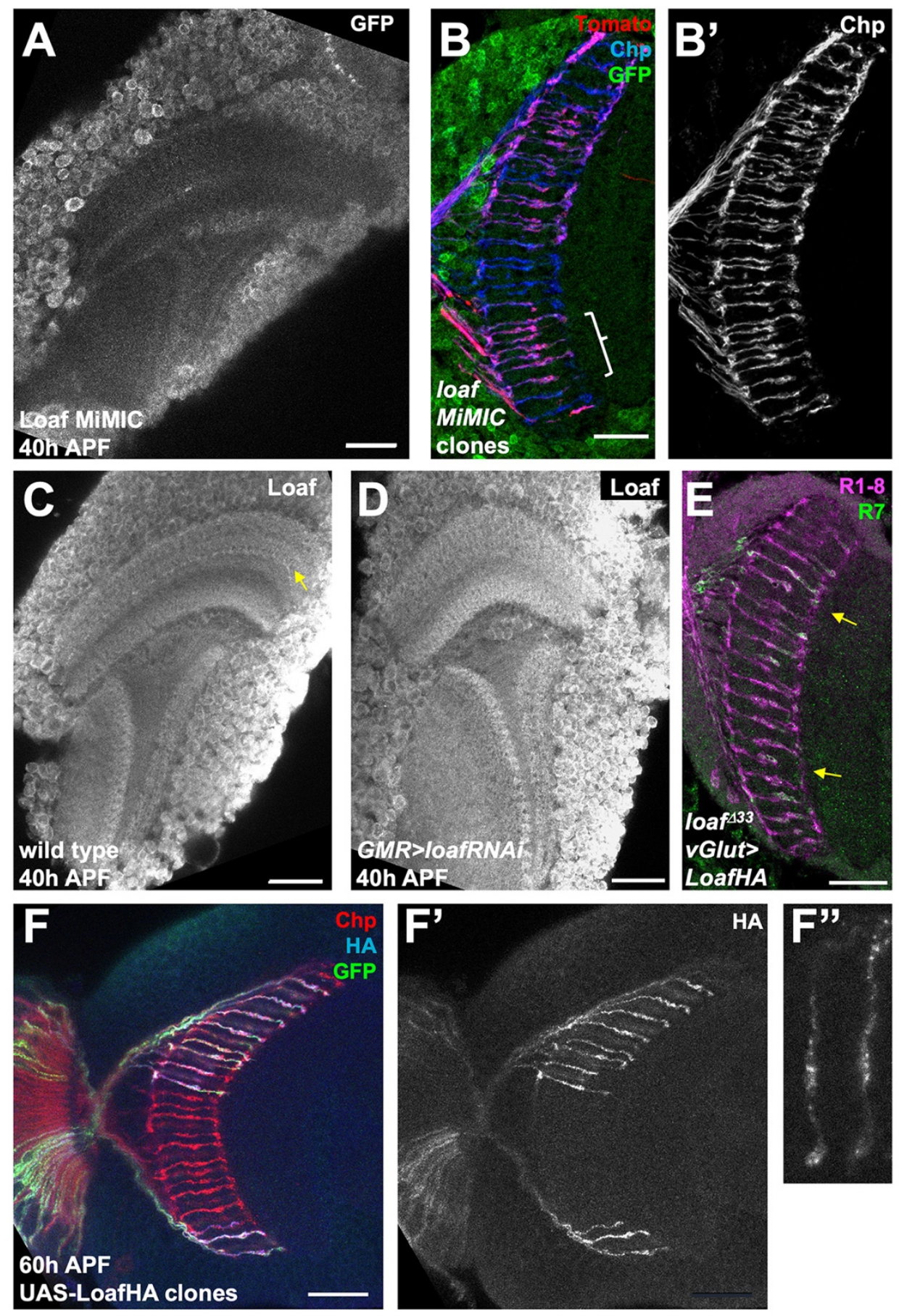

Douthit et al. Figure 4 - figure supplement 1 
Figure 4 - figure supplement 1: Loaf is expressed in many cells and enriched in R7 terminals. (A) Mi\{PT-GFSTF.1\}CG6024 ${ }^{\text {MIO0316-GFSTF.1 }}$ brain at $40 \mathrm{~h}$ APF stained for GFP. GFP-tagged Loaf is mostly confined to cell bodies and only weakly present in the neuropil. (B) cryostat section of an adult head in which Mi\{PT-GFSTF.1\}CG6024 MI00316-GFSTF.1 homozygous clones are marked with UAS-myr-tdTomato (red) and their axons are stained for Chp ( $\mathrm{B}^{\prime}$, blue in B). GFP-Loaf is stained in green. R7 mistargeting is observed in some clones homozygous for the insertion (bracket), indicating that it disrupts the function of the protein. (C, D) pupal brains at $40 \mathrm{~h}$ APF stained for Loaf. (C) wild type; (D) GMR-GAL4, UAS-dcr2; UAS-loaf RNAiBL. Loaf is enriched in R7 terminals at this stage (yellow arrow in C) and this staining is absent when loaf is knocked down in photoreceptors. (E) cryostat section of a vGlut-GAL4/UAS-LoafHA; loaf ${ }^{\triangle 33}$ adult head stained for Chp (magenta) and panR7-lacZ (green). Arrows indicate mistargeted R7 axons. (F) $60 \mathrm{~h}$ APF brain in which LoafHA is misexpressed in clones of photoreceptors with IGMR-GAL4, marked with GFP (green) and stained for Chp (red) and HA ( $F^{\prime}, F^{\prime \prime}$, blue in F). (F') shows an enlargement of two R7 axons. LoafHA is transported to R7 axons and terminals. Scale bars, $20 \mu \mathrm{m}$.

Loaf was expressed in glia with repo-Gal4, or in neuronal populations that expressed homothorax (hth)-GAL4, brain-specific homeobox (bsh)-GAL4, or Visual system homeobox (Vsx)GAL4 (Hasegawa et al. 2011; Li et al. 2013; Erclik et al. 2017) (Figure 4D). Expressing Loaf in photoreceptors with ey3.5-FLP, Act>CD2>GAL4 in a loaf mutant background likewise had no effect on R7 (Fig. 4D), indicating that the presence of Loaf in R7 when it is absent in other cells did not impede its targeting. However, we did observe a significant level of R7 mistargeting when Loaf was expressed in neurons that expressed apterous (ap)-GAL4, which is active from the third larval instar (Morante et al. 2011; Li et al. 2013) or in cholinergic neurons with Choline acetyltransferase (ChAT)-GAL4, which is active from mid-pupal stages (Meissner et al. 2019), in a loaf mutant background (Figure 4D-F). ap is expressed in the majority of cholinergic neurons in the medulla (Konstantinides et al. 2018), supporting the idea that cells in this population use Loaf to interact with R7. R7 targeting defects also occurred when Loaf was expressed in 
glutamatergic neurons in a loaf mutant background with Vesicular glutamate transporter (VGlut)-GAL4, which is active from early pupal stages (Meissner et al. 2019) (Figure 4D; Figure 4 - figure supplement 1E), indicating that more than one type of neuron interacts with R7 through Loaf.

\section{Loaf levels in the synaptic partners Tm5a/b and Dm9 influence R7 targeting}

The populations of cholinergic and glutamatergic neurons include the major synaptic targets of R7, suggesting the possibility that Loaf acts in these cells to influence their interactions with R7. The synapses that R7 forms with Dm8 also include the cholinergic output neurons Tm5a and Tm5b (Gao et al. 2008; Karuppudurai et al. 2014; Menon et al. 2019; Davis et al. 2020). To test the importance of Tm5a/b neurons we used GMR9D03-GAL4, which is expressed in a subset of these cells from early in development (Han et al. 2011)(Figure 5 figure supplement 1A, B) to express Loaf in a loaf mutant background. This again produced significant R7 mistargeting (Figure 5A, H), consistent with the hypothesis that Loaf levels in Tm5a/b influence R7. Although GMR9D03-GAL4 is also expressed in lamina neurons L2 and L3 (Akin et al. 2019), restoring Loaf only in lamina neurons with GH146-GAL4 (Schwabe et al. 2014) did not affect R7 (Figure 5H). Importantly, loaf mutant Tm5a/b cells did not have obvious morphological defects or cause R7 mistargeting (Figure 5D, E).

Among glutamatergic neurons, both Dm8 and Dm9 are synaptic partners of R7. Dm9 is a multicolumnar neuron that tracks R7 axons closely and mediates inhibition between neighboring ommatidia (Nern et al. 2015; Heath et al. 2020).The transcription factors Vestigial (Vg) and Defective proventriculus (Dve) are strongly enriched in Dm9 cells (Davis et al. 2020), and $d v e-G A L 4$ drives expression in Dm9 (Figure 5 - figure supplement 1C), although vg-GAL4 expression was not detectable in the adult brain. When used to express Loaf in a loaf mutant background, neither driver alone significantly affected R7 targeting, but the combination had a significant effect (Figure 5B, H), making Dm9 a candidate to provide Loaf that affects R7 targeting. Again, loaf mutant Dm9 cells and their presynaptic R7 axons appeared normal (Figure $5 F, G)$. Finally, we tested whether a contribution of Dm8 might be detectable in combination with other R7 synaptic target cells by restoring Loaf to loaf mutants with both ap-GAL4 and tj- 

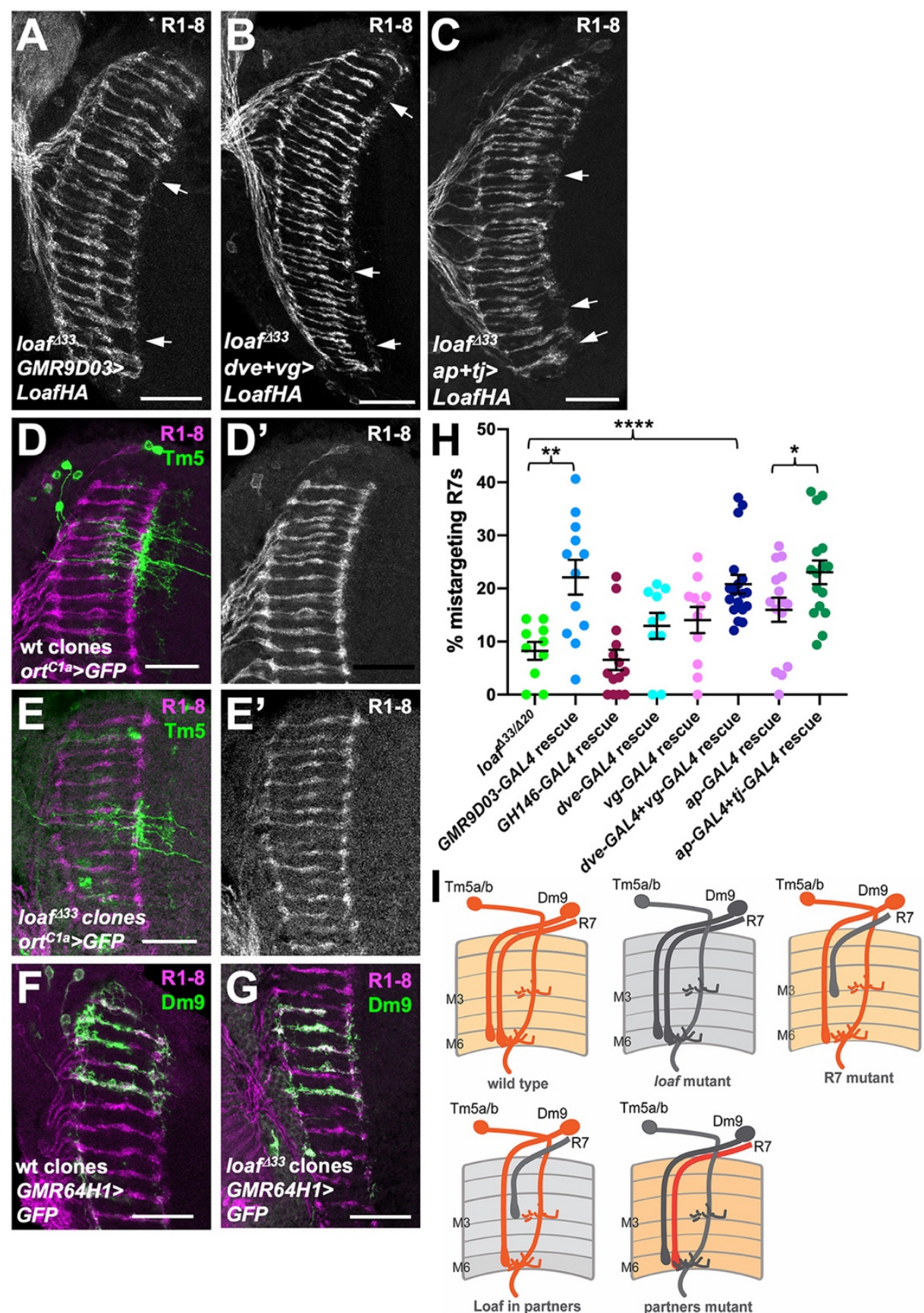

\section{Douthit et al. Figure 5}


Figure 5: Expressing Loaf in synaptic partners of $R 7$ in a loaf mutant causes mistargeting. (A-C) cryostat sections of adult heads stained for Chp. (A) UAS-LoafHA; GMR9D03-GAL4, loaf ${ }^{\triangle 33} /$ loaf $^{\Delta 33}$; (B) dve-GAL4, vg-GAL4/UAS-LoafHA; loaf $\triangle 33$; (C) ap-GAL4, tj-GAL4/UAS-LoafHA; loaf ${ }^{\Delta 33}$. Expressing Loaf in populations of neurons that form synapses with $\mathrm{R} 7$ in a loaf mutant background causes R7 mistargeting. (D-G) cryostat sections of adult heads in which clones generated with hs-FLP are labeled in green with UAS-CD8-GFP and R1-8 are stained with antiChp ( $D^{\prime}, E^{\prime}$, magenta in D-G). (D) wild type and (E) loaf ${ }^{\Delta 33}$ mutant clones in which Tm5a/b/c and Tm20 are labeled with ort ${ }^{C 1 a}-G A L 4$. The genotypes are (D) hsFLP, UAS-GFP; ortc1a-GAL4/CyO; FRT80/tub-GAL80, FRT80; (E) hsFLP, UAS-GFP; ortc1a-GAL4/CyO; loaf ${ }^{\Delta 33}$, FRT80/tub-GAL80, FRT80. (F) wild type and (G) loaf ${ }^{\Delta 33}$ mutant clones in which Dm9 cells are labeled with GMR64H1-GAL4. The genotypes are (F) hsFLP, UAS-GFP; GMR64H1-GAL4, FRT80/tub-GAL80, FRT 80; (G) hsFLP, UAS-GFP; GMR64H1-GAL4, loaf ${ }^{433}$, FRT80/tub-GAL80, FRT 80. The morphologies of wild type and loaf mutant Tm5 and Dm9 cells appear similar. (H) quantification of the percentage of R7 axons that failed to reach the M6 layer in the indicated genotypes. $\mathrm{n}=10$ (loaf ${ }^{\Delta 33} /$ loaf $^{\Delta 20}$; dve-GAL4 rescue), 12 (GMR9D03-GAL4 rescue), 14 (GH146-GAL4 rescue), 11 ( $v g-G A L 4$ rescue), 18 (dve-GAL4 + vg-GAL4 rescue), 15 (ap-GAL4 rescue), 16 (ap-GAL4 + tjGAL4 rescue). Error bars show mean \pm SEM. *,$p<0.05 ; * *, p<0.01 ; * * * *, p<0.0001$ by unpaired t-test. (I) model showing that the presence of Loaf in Dm9 or Tm5a/b when loaf is absent in R7 causes R7 mistargeting. Scale bars, $20 \mu \mathrm{m}$. 

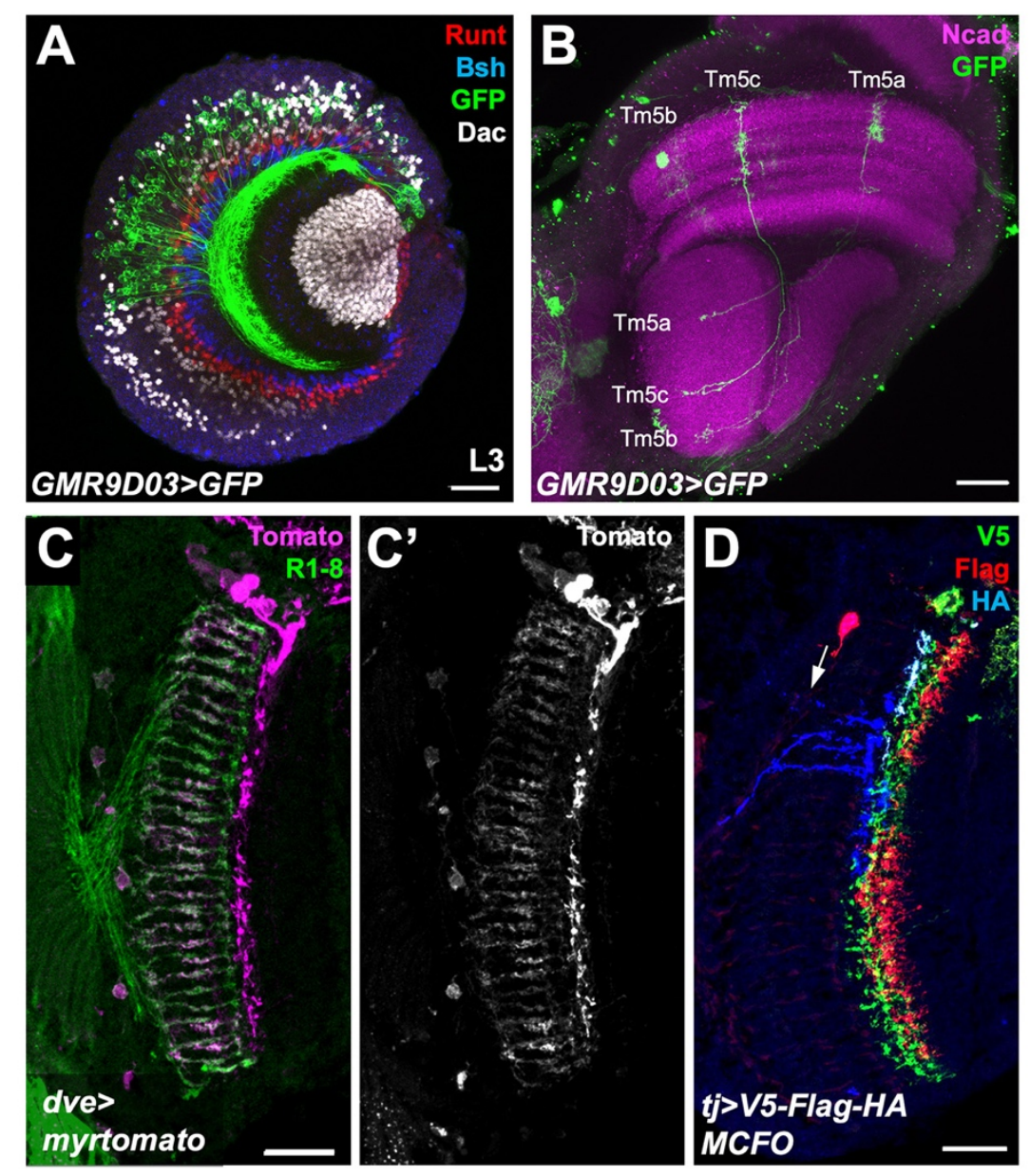

Figure 5 - figure supplement 1: GAL4 drivers for Tm5a/b and Dm9. (A) a third instar larval brain expressing UAS-CD8-GFP with GMR9D03-GAL4, stained for GFP (green), Dac (white), Runt (red) and Bsh (blue). GMR9D03-GAL4-expressing cell bodies do not express Bsh, Runt or Dac, consistent with Tm5a/b identity, and are restricted to the dorsal half of the medulla cortex. (B) adult brain expressing UAS>stop>CD4-tdGFP with GMR9D03-GAL4 after a 7-minute heat shock at late L3 to induce the expression of hs-FLP2PEST, stained for GFP (green) and Ncad (magenta). Cells with the morphology and projection pattern of $\mathrm{Tm} 5 \mathrm{a} / \mathrm{b} / \mathrm{c}$ are labeled with GFP, showing that the GMR9D03 enhancer is expressed in these cell types. (C) cryostat section of adult head expressing UAS-myr-tdTomato with $d v e^{N P 3428}-G A L 4$, stained for Tomato ( $C^{\prime}$, magenta in $C$ ) and Chp (green). $d v e^{N P 3428}-G A L 4$ labels cells with the morphology and projection pattern of Dm9. (D) tj-GAL4 expressing cells labeled with V5 (green), FLAG (red) and HA (blue) using the MultiColor FlpOut (MCFO) system. The cell labeled in blue (arrow) resembles a Dm11 cell. Scale bars, 20 $\mu \mathrm{m}$. 

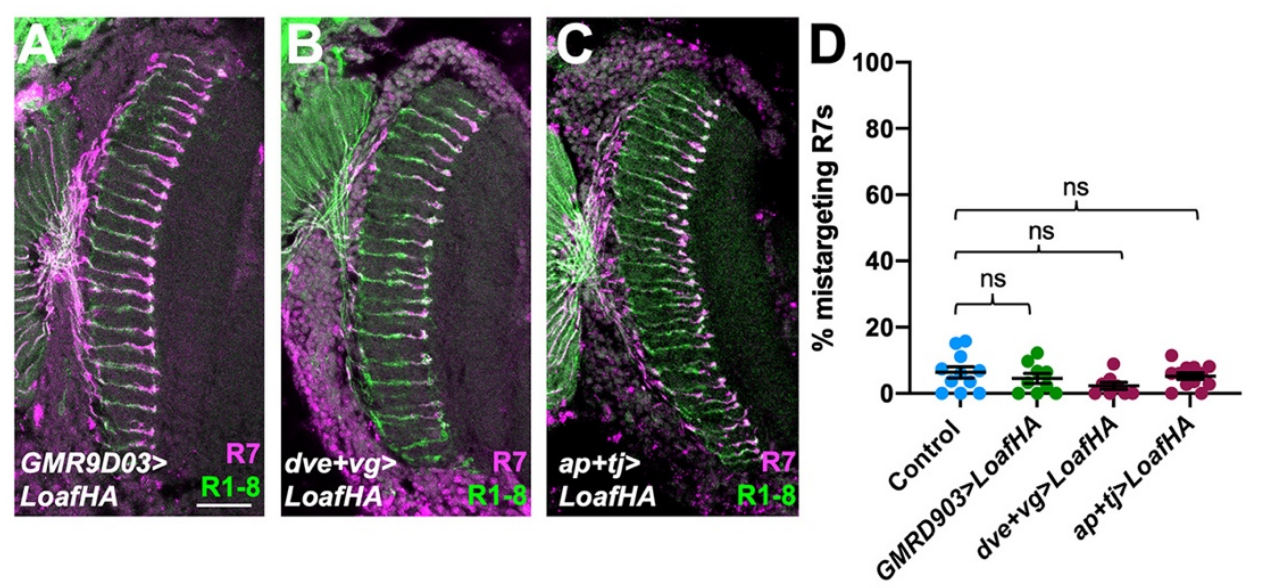

Figure 5 - figure supplement 2: Overexpression of Loaf in the synaptic partners of R7 does not cause mistargeting. (A-C) cryostat sections of adult heads stained for Chp (green) and panR7lacZ (magenta). (A) GMR9D03-GAL4; UAS-LoafHA; (B) dve-GAL4, vg-GAL4; UAS-LoafHA; (C) apGAL4, tj-GAL4; UAS-LoafHA. Overexpression of Loaf in these cell types does not cause significant mistargeting. Scale bar, $20 \mu \mathrm{m}$. (D) quantification of the percentage of R7 axons that failed to reach the M6 layer in these genotypes. $n=11$ (control), 9 (GMR9D03>LoafHA), 3 $(d v e+v g>$ LoafHA) or $12(a p+t j>$ LoafHA). ns, not significant by unpaired t-test.

GAL4. This produced significantly more R7 mistargeting than ap-GAL4 alone (Figure $5 \mathrm{C}, \mathrm{H}$ ), suggesting that Dm8 or another tj-GAL4 expressing neuron such as Dm11 (Courgeon and Desplan 2019), which also projects to the M6 layer (Nern et al. 2015) (Figure 5 - figure supplement 1D), may contribute to the pool of Loaf that influences R7 targeting. However, $\mathrm{Tm} 5 \mathrm{a} / \mathrm{b}$ and Dm9 appear to play a more significant role (Figure 5I). Overexpressing Loaf with the GMR9D03-GAL4, dve-GAL4 and vg-GAL4, or ap-GAL4 and tj-GAL4 drivers in a wild-type background did not cause R7 mistargeting (Figure 5 - figure supplement 2); because Loaf is normally enriched in R7 terminals, it is possible that the Loaf levels produced in the processes of synaptic partner cells in these overexpression experiments did not exceed those present in R7. 


\section{Loaf may act indirectly through cell-surface molecules}

To determine whether Loaf could function as a homophilic cell adhesion molecule, we transfected HA-tagged Loaf into S2 cells and conducted cell aggregation assays (Ting et al. 2005; Astigarraga et al. 2018). We did not observe significant aggregation of the transfected cells, although the positive control Sidekick (Sdk) (Astigarraga et al. 2018) induced aggregation under the same conditions (Figure 6A, B, Figure 6 - figure supplement 1A). Unlike Sdk, neither tagged nor untagged Loaf showed strong localization to the plasma membrane; most Loaf was present in punctate structures inside the cells (Figure 6C-E). These structures showed partial colocalization with Hepatocyte growth factor-regulated tyrosine kinase substrate (Hrs) and Rab7 (Figure 6D, E), two markers of late endosomes, but did not colocalize with the recycling endosome marker Rab11-GFP (Figure 6 - figure supplement $1 \mathrm{C}$ ). When expressed in the retina in vivo, LoafHA also partially colocalized with Rab7 and Hrs, but not with the lysosomal markers ADP-ribosylation factor-like 8 (Arl8) or Vacuolar $\mathrm{H}^{+}$-ATPase 55kD subunit (Vha55) (Figure 6F, G, J), and untagged Loaf again showed a similar localization (Figure 6 - figure supplement 1D). In clones of cells mutant for the ESCRT complex component Tumor susceptibility gene 101 (TSG101), endocytosed proteins such as Notch accumulate in late endosomes (Moberg et al. 2005), and we found that LoafHA colocalized with Notch (Figure 6 - figure supplement 1E), confirming its presence in the endocytic pathway.

GFP-tagged endogenous Loaf appeared to localize to the cytoplasm of all photoreceptors, but unlike overexpressed Loaf, it was primarily found close to the plasma membrane rather than in late endosomes (Figure 6-figure supplement 1B); as noted above, this tag disrupts the function of the Loaf protein. As a more stringent test of whether Loaf ever reaches the plasma membrane, we transfected S2 cells with a form of Loaf tagged at its extracellular N-terminus with the V5 epitope, and incubated live cells with antibodies to V5. No staining was observed in these conditions (Figure $6 \mathrm{H}$ ). As controls for this experiment, V5 staining was detected in cells that were fixed and permeabilized, and antibodies to HA detected cotransfected HASdk on the surface of live cells as well as in vesicles internalized during the incubation (Figure $6 \mathrm{H}, \mathrm{I}$ ). These results suggest that Loaf is not itself a cell surface adhesion 

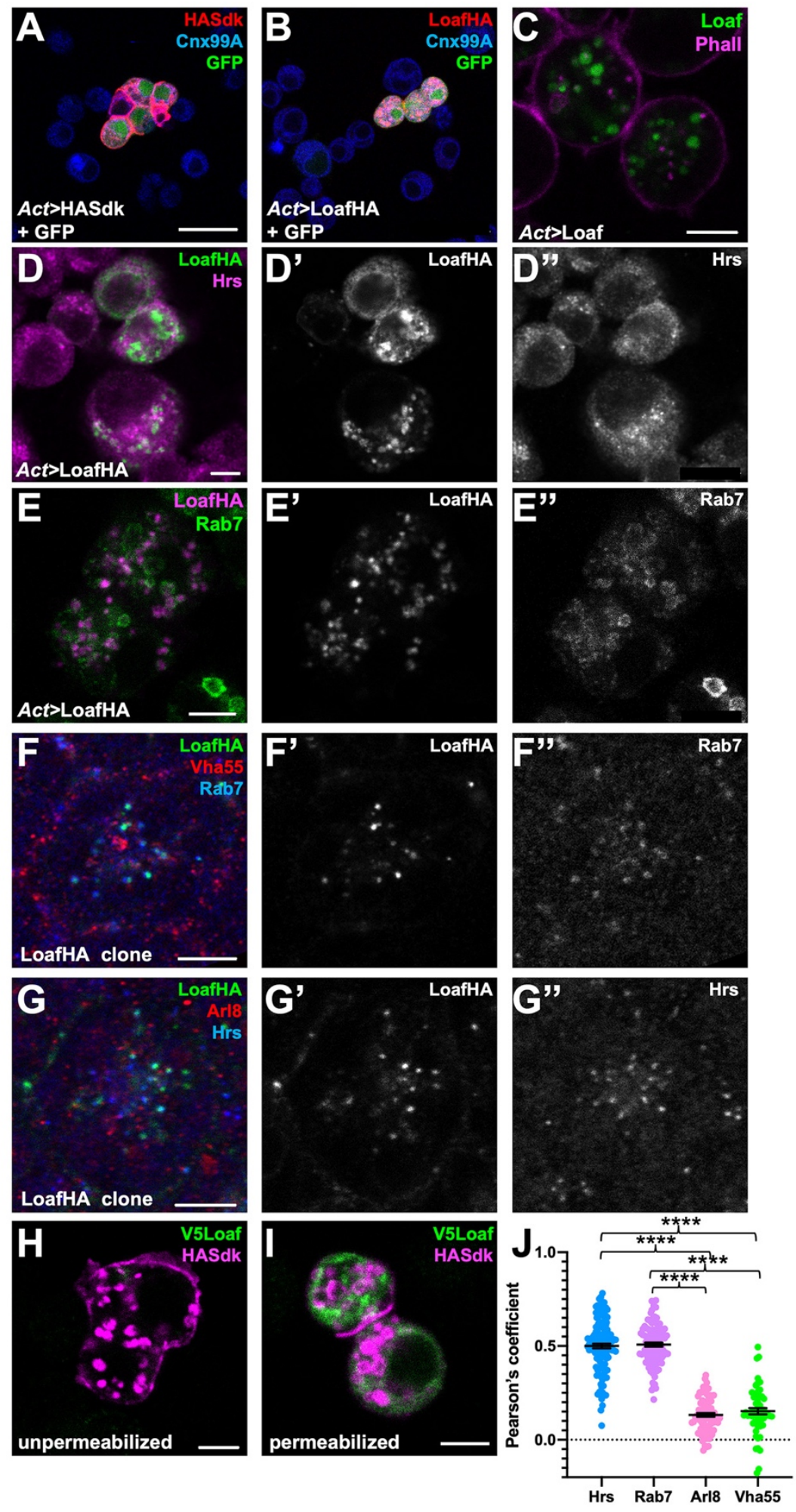

Douthit et al. Figure 6 
Figure 6: Loaf localizes to endosomes. (A, B) S2 cells transfected with Act-GAL4, UAS-GFP and UAS-HASdk (A) or UAS-LoafHA (B) and allowed to aggregate, stained for GFP (green), HA (red) and the ER marker Calnexin 99A (Cnx99A, blue). Sdk localizes to cell contacts and induces aggregation, but Loaf does not. (C) S2 cells transfected with Act-GAL4 and UAS-Loaf, stained with anti-Loaf (green) and Phalloidin (magenta). (D, E) S2 cells transfected with Act-GAL4 and UAS-LoafHA, stained for HA ( $D^{\prime}, E^{\prime}$, green in $D$, magenta in $\left.E\right)$, Hrs ( $D^{\prime \prime}$, magenta in $\left.D\right)$, or Rab7 $\left(E^{\prime \prime}\right.$, green in E). Loaf localizes to intracellular vesicles that show some colocalization with Hrs and Rab7. (F, G) ommatidia from $42 \mathrm{~h}$ APF pupal retinas in clones expressing UAS-LoafHA, stained for HA ( $F^{\prime}, G^{\prime}$, green in $\left.F, G\right)$ Rab7 (F', blue in F), Vha55 (red in F), Hrs (G', blue in $G$ ) and Arl8 (red in G). Loaf colocalizes with the endosomal markers Rab7 and Hrs, but not the lysosomal markers Vha55 and Arl8, in photoreceptors in vivo. $(\mathrm{H}, \mathrm{I}) \mathrm{S} 2$ cells transfected with Act-GAL4, UAS-HASdk and UAS-V5Loaf and incubated with antibodies to HA (magenta) and V5 (green) at room temperature prior to fixation (H) or after fixation and permeabilization (I). Sdk is detected on the cell surface and in internalized vesicles without fixation, but Loaf is not. (J) quantification of the colocalization of LoafHA with Hrs, Rab7, Arl8 and Vha55 in $42 \mathrm{~h}$ APF retinas by Pearson's correlation. $\mathrm{n}=131$ ommatidia from 19 retinas (Hrs), 100 ommatidia from 19 retinas (Rab7), 85 ommatidia from 16 retinas (Arl8) or 59 ommatidia from 11 retinas (Vha55). Error bars show mean \pm SEM. ${ }^{* * * *}, \mathrm{p}<0.0001$. Scale bars, $20 \mu \mathrm{m}(\mathrm{A})$ or $5 \mu \mathrm{m}(\mathrm{C}-\mathrm{I})$. 

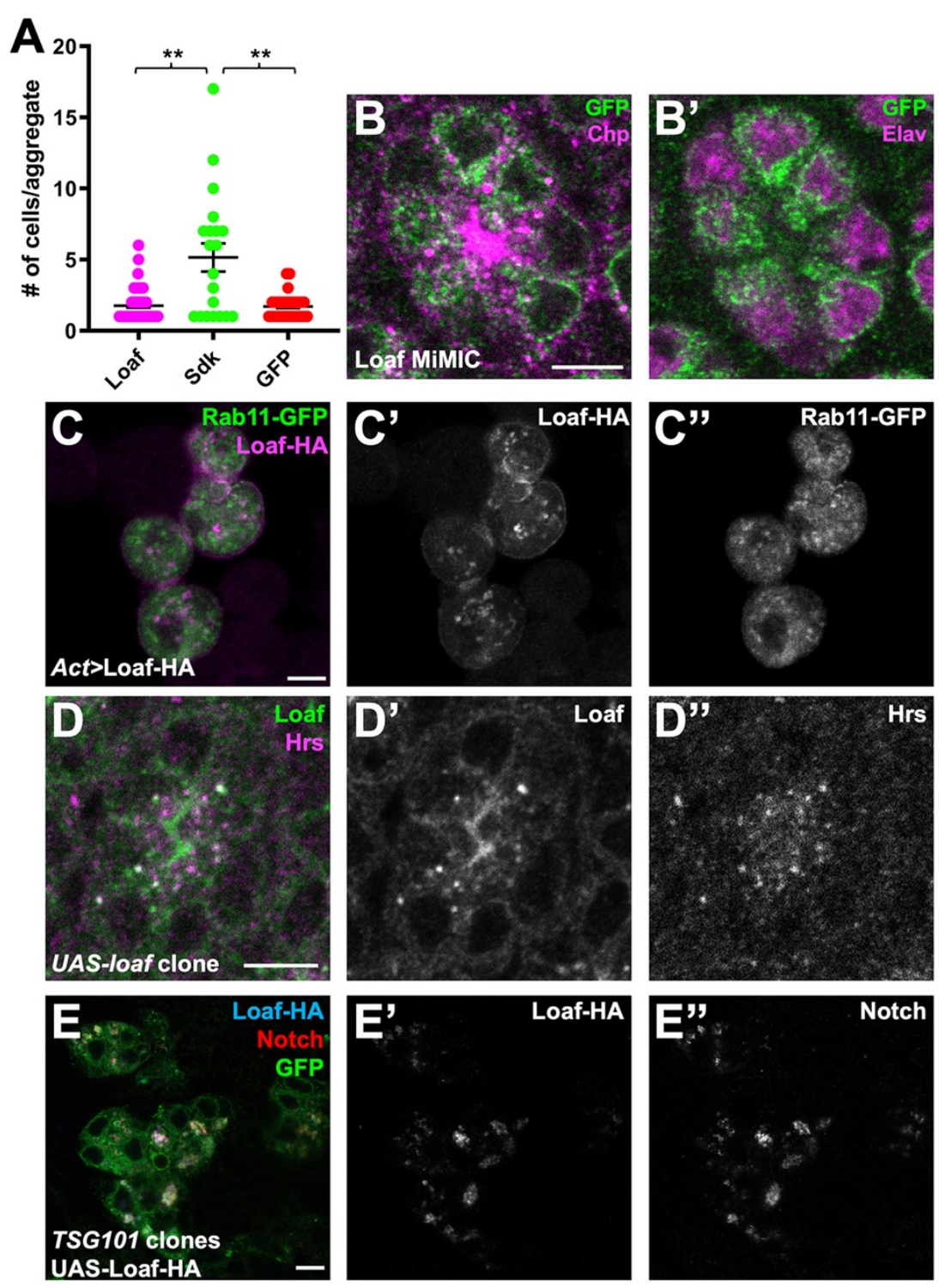

Douthit et al. Figure 6 - figure supplement 1 
Figure 6 - figure supplement 1: Loaf localizes to endosomes and does not mediate cell aggregation. (A) quantification of the number of cells per aggregate for S2 cells transfected with Act-GAL4 and UAS-LoafHA, UAS-HASdk, or UAS-GFP. $\mathrm{n}=63$ (Loaf), 20 (Sdk) or 27 (GFP). **, $\mathrm{p}<0.01$ by unpaired t-test with Welch's correction. (B) ommatidium from a Loaf protein trap

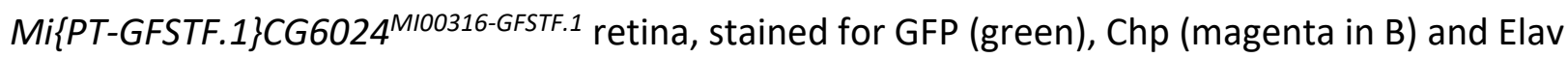
(magenta in $\mathrm{B}^{\prime}$ ). Endogenously tagged LoafGFP is present just inside the plasma membrane marked by Chp and in the cytoplasm close to the rhabdomere. (C) S2 cells transfected with ActGAL4, UAS-LoafHA and UAS-Rab11-GFP, stained for HA ( $C^{\prime}$, magenta in C) and GFP ( $C^{\prime \prime}$, green in C). Loaf does not colocalize with Rab11. (D) ommatidium from a clone expressing UAS-Loaf in a $42 \mathrm{~h}$ APF retina, stained for Loaf ( $D^{\prime}$, green in D) and Hrs ( $D^{\prime \prime}$, magenta in D). Untagged Loaf colocalizes with Hrs in vivo. (E) $42 \mathrm{~h}$ APF retina with a TSG101 clone in which UAS-LoafHA was expressed with IGMR-GA/4, positively labeled with GFP and stained for GFP (green), HA (E', blue in $E)$ and Notch ( $E^{\prime \prime}$, red in E). Loss of TSG101 causes accumulation of enlarged endosomes in which Loaf colocalizes with internalized Notch. Scale bars, $5 \mu \mathrm{m}$.

molecule, but could regulate the trafficking or cell surface localization of proteins involved in cell adhesion or synapse formation.

\section{Loaf interacts with Lar and enhances the function of Lrp4}

We next searched for candidate proteins that might be regulated by Loaf. One possibility we considered was Lar, an RPTP that is required for normal R7 targeting (Clandinin et al. 2001; Maurel-Zaffran et al. 2001; Hofmeyer and Treisman 2009). Lar acts in R7 and not the target region (Clandinin et al. 2001; Maurel-Zaffran et al. 2001), so its ligand, which remains unknown, would also have to be regulated by Loaf to account for the effect of Loaf in synaptic partners of R7. To test for a genetic interaction between loaf and Lar, we knocked down these genes using the photoreceptor driver long GMR-GAL4 (IGMR-GAL4) (Wernet et al. 2003). Expression of either loaf RNAi or Lar RNAi with this driver affected only a subset of R7 axons, 
but simultaneous expression of both RNAi lines had a synergistic effect, causing almost all R7 axons to terminate in the M3 layer (Figure 7A-D). This suggests that Loaf and Lar are involved in the same process. Similarly, loaf knockdown enhanced the mistargeting phenotype of mutations in the downstream gene Liprin- $\alpha$, although this effect could simply be additive (Figure 7 - figure supplement 1G-J). Overexpression of Lar in photoreceptors, either alone or together with Loaf, did not cause any significant defects (Figure 7- figure supplement 1A, B, D). However, overexpression of Lar in loaf mutant photoreceptors could rescue R7 targeting (Figure 7- figure supplement 1C, D), indicating that Lar can compensate for the lack of loaf and is thus unlikely to be its primary effector. Consistent with this conclusion, we found that loaf was not required for HA-tagged Lar to be transported into photoreceptor axons (Figure 7 figure supplement $1 \mathrm{E}, \mathrm{F})$.

We also investigated LDL receptor related protein 4 (Lrp4), based on its role as a presynaptic organizer in the olfactory system (Mosca et al. 2017), its postsynaptic signaling function at the vertebrate neuromuscular junction (Yumoto et al. 2012), and the requirement for chaperones to promote the trafficking of other LDL family members (Culi et al. 2004; Wagner et al. 2013). We found evidence that the level of Lrp4 can affect R7 targeting and that its effect on R7 is regulated by Loaf. Overexpressing Lrp4 in photoreceptors caused R7 axons to contact each other or hyperfasciculate either in the M3 or M6 layers of the medulla (Figure 7E, $\mathrm{H})$. These defects were more severe, and included overshooting of the M6 layer by some R7 axons, when Lrp4 was coexpressed with Loaf (Figure 7F, H), but were almost absent when Lrp4 was expressed in loaf mutant photoreceptors (Figure 7G, H). Lrp4 overexpression also resulted in abnormal numbers and arrangements of cone and pigment cells in the retina (Figure 7 figure supplement 2A). Again, these defects were more severe when Lrp4 was coexpressed with Loaf, and were not observed when Lrp4 was expressed in loaf mutant cells (Figure 7 - figure supplement 2B, C). Although Lrp4HA had a more granular appearance in loaf mutant than in wild type photoreceptor cell bodies (Figure 7 - figure supplement 2D, F), it was still transported into their axons (Figure 7 - figure supplement 2E, G), and its level of expression appeared unaffected (Figure 7 - figure supplement $2 \mathrm{H}$ ). 

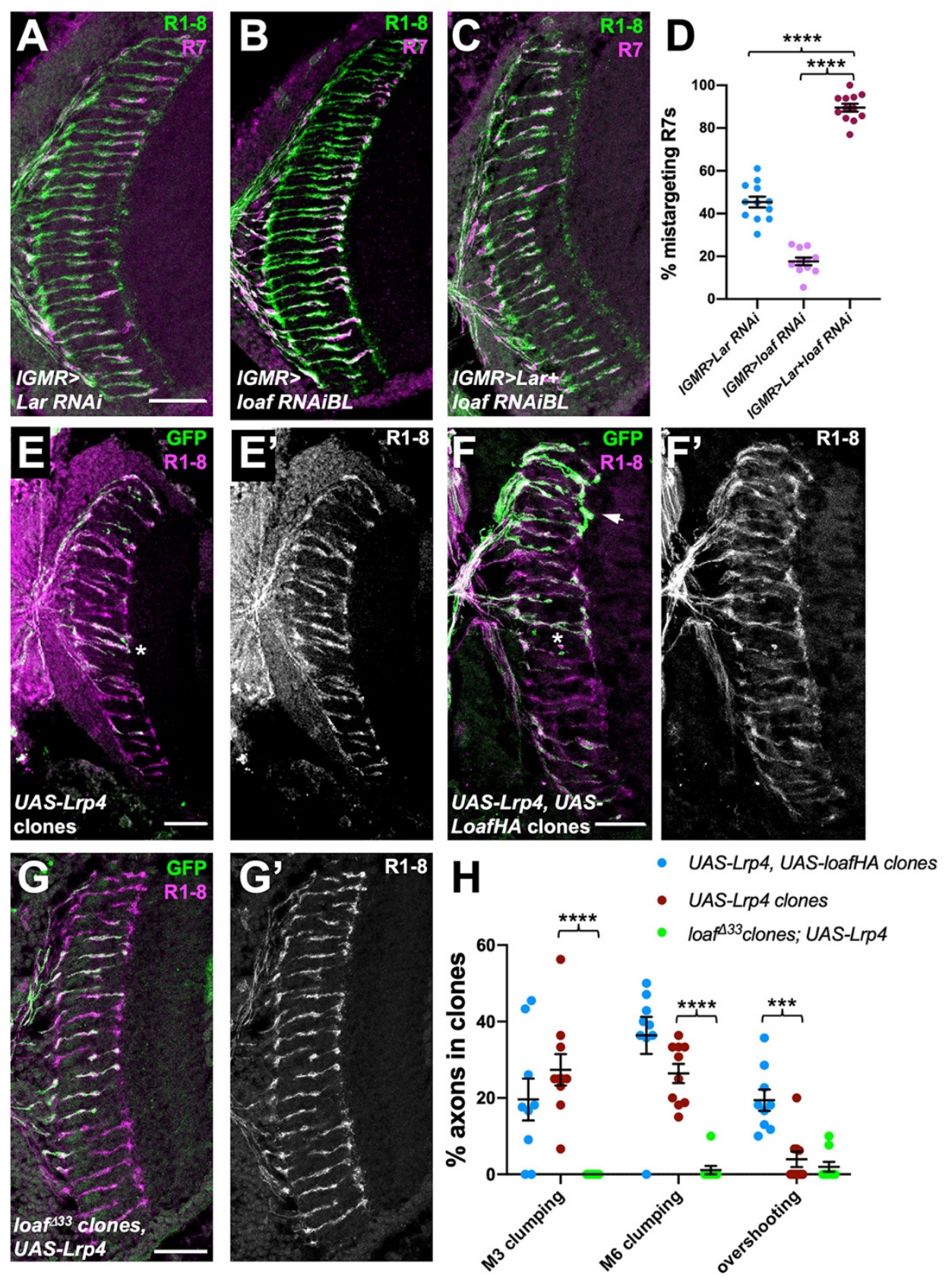

Douthit et al. Figure 7 
Figure 7: loaf genetically interacts with Lar and Lrp4. (A-C) cryostat sections of adult heads stained for Chp (green) and panR7-lacZ (magenta). (A) IGMR-GAL4, UAS-dcr2/UAS-Lar RNAi; (B) IGMR-GAL4, UAS-dcr2; UAS-Ioaf RNAiBL; (C) IGMR-GAL4, UAS-dcr2/UAS-Lar RNAi; UAS-loaf RNAiBL. With this driver, Lar RNAi induces moderate and loaf RNAi mild R7 mistargeting, but the combination has a severe phenotype. (D) quantification of the percentage of R7 axons that failed to reach the M6 layer in the indicated genotypes. $\mathrm{n}=12$ (Lar RNAi, Lar RNAi + loaf RNAi) or 11 (loaf RNAi). ****, $\mathrm{p}<0.0001$ by unpaired t-test. (E-G) cryostat sections of adult heads with clones positively labeled with GFP, stained for Chp ( $E^{\prime}, F^{\prime}, G^{\prime}$, magenta in E-G) and GFP (green). (E) clones expressing UAS-Lrp4 with IGMR-GAL4. (F) clones expressing UAS-Lrp4 and UASLoafHA with IGMR-GAL4. (C) loaf ${ }^{\triangle 33}$ clones in which UAS-Lrp4 is expressed with IGMR-GAL4. (H) quantification of the percentage of labeled R7s of each genotype that show R7 axons clumping together in the M3 (asterisk in F) or M6 (asterisk in E) layers or overshooting the M6 layer (arrow in F). $\mathrm{n}=9$ heads (UAS-Lrp4, UASLoafHA; loaf ${ }^{433}$ clones, UAS-Lrp4) or 10 (UAS-Lrp4). Error bars show mean \pm SEM. ${ }^{* * *}, \mathrm{p}<0.001 ; * * * *, \mathrm{p}<0.0001$ by multiple t-tests with two-stage linear step-up procedure. Scale bars, $20 \mu \mathrm{m}$. 

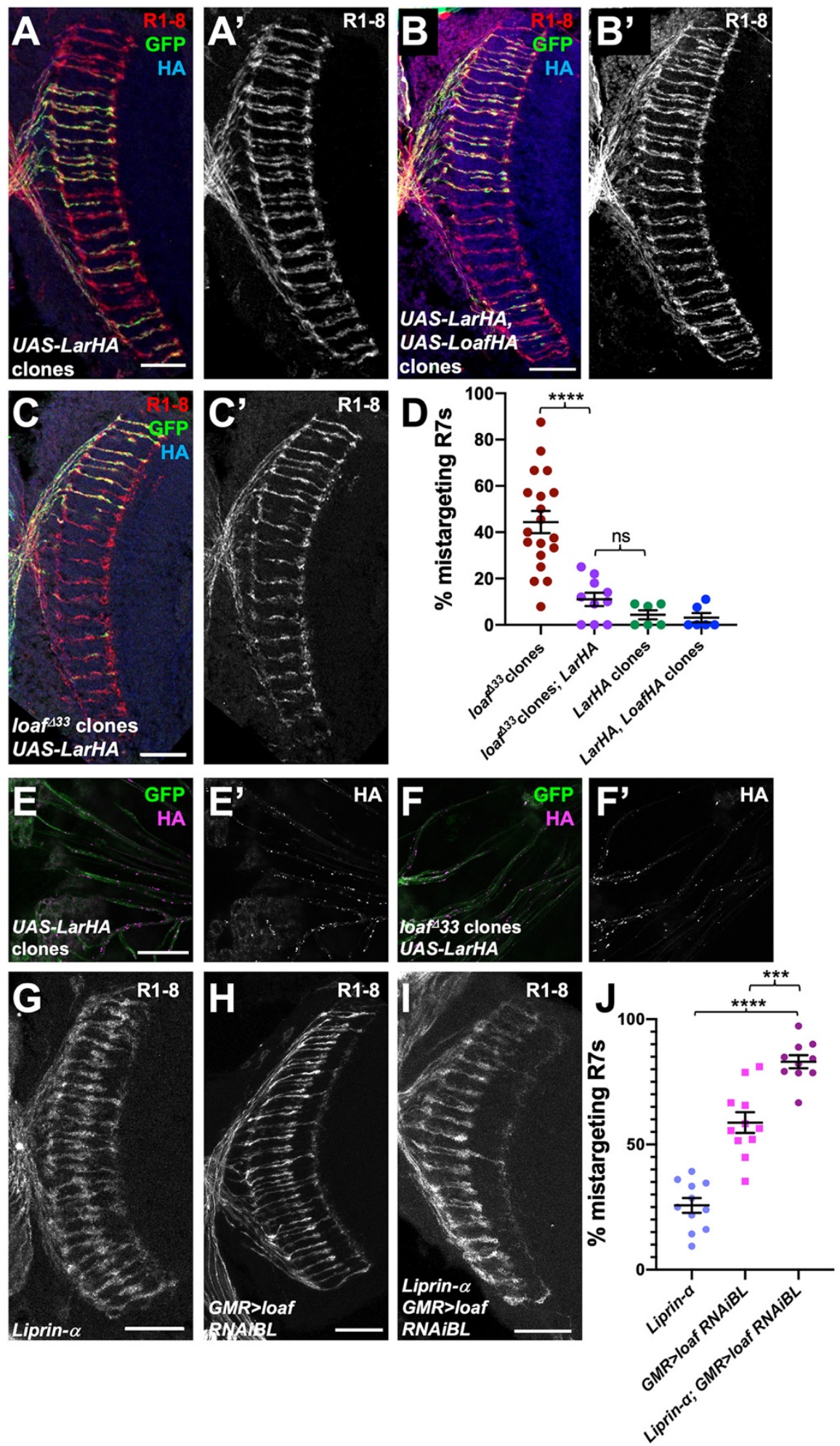

Douthit et al. Figure 7 - figure supplement 1 
Figure 7 - figure supplement 1: Lar overexpression can compensate for loss of Loaf. (A-C) cryostat sections of adult heads with clones positively labeled with GFP, stained for Chp ( $A^{\prime}$, $B^{\prime}$, $\mathrm{C}^{\prime}$, red in A-C), GFP (green) and HA (blue). (A) clones expressing UAS-LarHA with IGMR-GAL4. (B) clones expressing UAS-LarHA and UAS-LoafHA with IGMR-GAL4. (C) loaf ${ }^{433}$ clones in which UASLarHA is expressed with IGMR-GAL4. (D) quantification of the percentage of R7 axons that failed to reach the M6 layer in the indicated genotypes. Expressing Lar rescues mistargeting in loaf mutant clones. $\mathrm{n}=19$ (loaf ${ }^{\Delta 33}$ clones), 10 (loaf ${ }^{\Delta 33}$ clones; LarHA), or 6 (LarHA clones; LarHA, LoafHA clones). Error bars show mean \pm SEM. $* * * *, p<0.0001$; ns, not significant by unpaired $t-$ test with Welch's correction. (E, F) photoreceptor axons from clones in $42 \mathrm{~h}$ APF retinas positively labeled with GFP, stained for GFP (green) and $H A\left(E^{\prime}, F^{\prime}\right.$, magenta in $\left.E, F\right)$. (E) wild type clones expressing UAS-LarHA with IGMR-GAL4; (F) loaf ${ }^{\triangle 33}$ clones in which UAS-LarHA is expressed with IGMR-GAL4. Lar is transported into axons in wild type or loaf mutant photoreceptors. (G-I) cryostat sections of adult heads stained for Chp. (G) Liprin- $\alpha^{\text {oos; }}$ (H) GMRGAL4/UAS-IoafRNAiBL; (I) Liprin- $\alpha^{o o s}$; GMR-GAL4/UAS-IoafRNAiBL. (J) quantification of the percentage of $\mathrm{R} 7$ axons that failed to reach the $M 6$ layer in the indicated genotypes. $n=11$ (Liprin- $\alpha, G M R>$ loafRNAiBL) or 10 (Liprin- $\alpha$; GMR>loafRNAiBL). ****, $\mathrm{p}<0.0001 ; * * *, \mathrm{p}=0.0001$. Scale bars, $20 \mu \mathrm{m}$.

Despite the effect of Loaf on Lrp4 function, Lrp4 is unlikely to fully explain the effects of loaf on R7 targeting, as R7 axons projected normally in Lrp4 mutant clones (Figure 7 - figure supplement 2I). Moreover, expressing loaf RNAi in photoreceptors resulted in R7 mistargeting even in an Lrp4 null mutant background (Figure 7 - figure supplement 2J, K). These results show that Loaf can affect the function of cell surface proteins, and suggest that it could act by regulating Lrp4 and/or other cell surface molecules that act as a readout of its levels to control the interactions between $\mathrm{R} 7$ and its postsynaptic partners. 

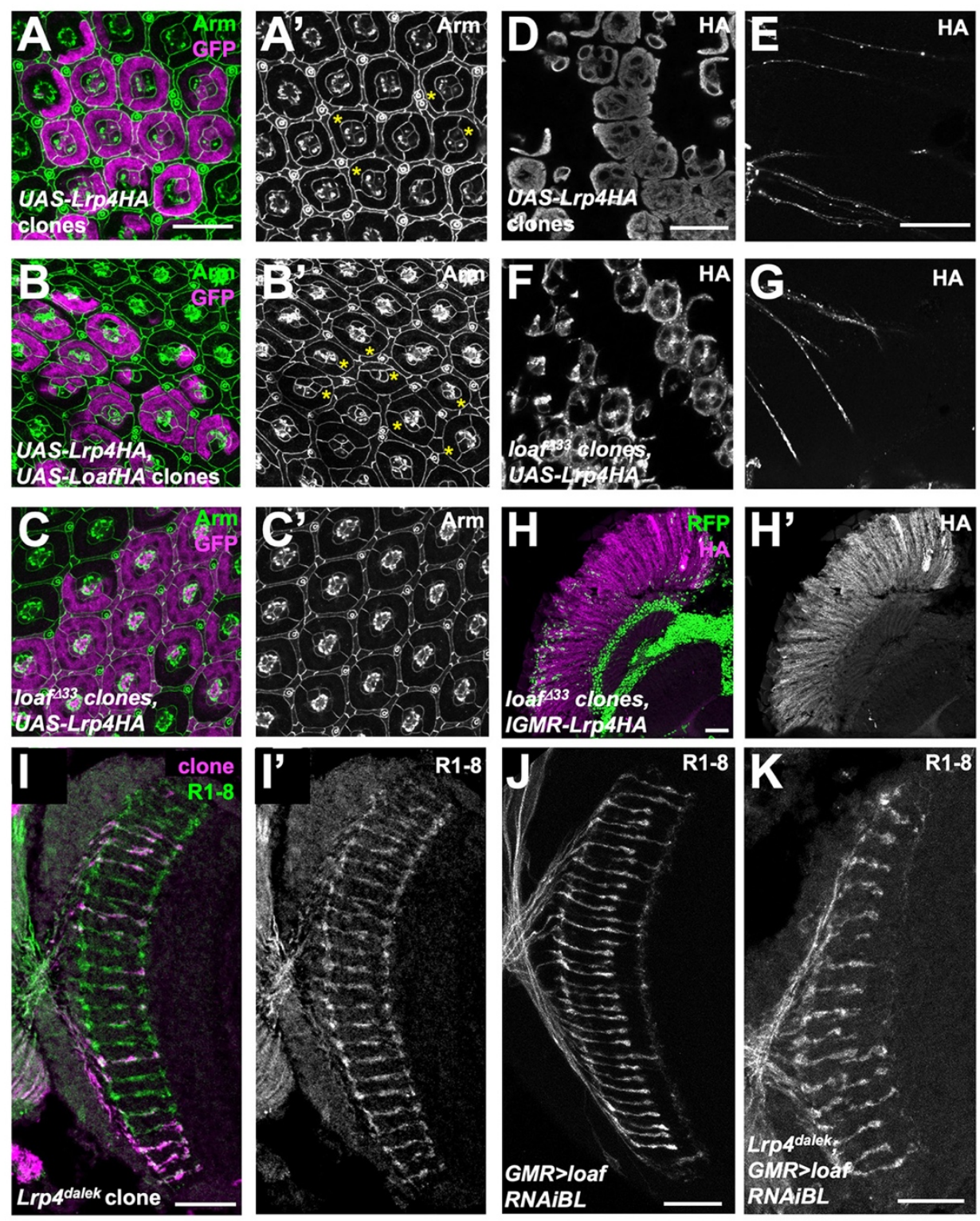

Douthit et al. Figure 7 - figure supplement 2 
Figure 7 - figure supplement 2: Lrp4 is not the only effector of Loaf. (A-C) 42 h APF retinas in which clones are positively labeled with GFP, stained for GFP (magenta) and Arm ( $A^{\prime}-C^{\prime}$, green in A-C). (A) clones expressing UAS-Lrp4 with IGMR-GAL4; (B) clones expressing UAS-Lrp4 and UAS-LoafHA with IGMR-GAL4; (C) loaf $f^{\triangle 33}$ clones in which UAS-Lrp4HA is expressed with IGMRGAL4. The ordered lattice structure of cone and pigment cells at the apical surface of the retina shows abnormalities (asterisks) when Lrp4 is overexpressed. These are more severe with Loaf coexpression and rescued in loaf mutant clones. (D-G) 42h APF pupal retinas in which UASLrp4HA is expressed with IGMR-GAL4 in wild type clones (D, E) or loaf ${ }^{\Delta 33}$ mutant clones $(F, G)$, stained for HA. $(D, F)$ show photoreceptor cell bodies and $(E, G)$ show their axons. Lrp4 is still transported into axons in loaf mutant clones, but has a different appearance in cell bodies. $(H)$ cryostat section of an adult head in which UAS-Lrp4HA is expressed in all cells with IGMR-GAL4, stained for $\mathrm{HA}\left(\mathrm{H}^{\prime}\right.$, magenta in $\left.\mathrm{H}\right)$. loaf ${ }^{\triangle 33}$ clones are marked by the absence of nuclear RFP (green). The level of Lrp4 protein is unaffected by loss of loaf. (I-K) cryostat sections of adult heads stained for Chp (I', J, K, green in I) and GFP to positively label clones (magenta in I). (I) Lrp4 ${ }^{\text {dalek }}$ clones do not show R7 mistargeting. (J, K) GMR-GAL4 driving UAS-loaf RNAiBL causes $\mathrm{R} 7$ mistargeting in an otherwise wild type background $(\mathrm{J})$ and in an Lrp4 ${ }^{\text {dalek }}$ background (K). Scale bars, $20 \mu \mathrm{m}$. 


\section{Discussion}

\section{Tm5a/b and Dm9 cells provide cues for $\mathrm{R} 7$ targeting}

The layered arrangement of neuronal processes in the medulla makes R7 axon targeting a sensitive model system in which to elucidate how growth cones select the correct postsynaptic partners. However, it has not been clear which cells are responsible for retaining R7 axons in the M6 layer. The RPTP Lar, which forms a hub for the assembly of presynaptic structures through the adaptor protein Liprin- $\alpha$ (Choe et al. 2006; Hofmeyer et al. 2006; Takahashi and Craig 2013; Bomkamp et al. 2019), acts in R7 to stabilize filopodia in the M6 layer by promoting synapse formation (Clandinin et al. 2001; Maurel-Zaffran et al. 2001; Ozel et al. 2019). Our findings that Lar and loaf show a strong genetic interaction and that Lar overexpression can rescue the loss of loaf suggest that like Lar, Loaf stabilizes synaptic contacts. Although Lar family RPTPs can recognize a variety of ligands (Han et al. 2016), the ligand involved in R7 targeting and its cellular source remain unknown (Hofmeyer and Treisman 2009). One candidate is Ncad, which is required at an early stage of development in both R7 and medulla neurons, but its widespread expression has made it difficult to determine in which neurons it acts to promote R7 synapse stabilization (Lee et al. 2001; Ting et al. 2005; Yonekura et al. 2007; Ozel et al. 2015).

R7 cells form numerous synapses with Dm8 interneurons, which are essential for ultraviolet spectral preference (Gao et al. 2008; Takemura et al. 2013) and fall into two classes that are postsynaptic to either yR7 or pR7 cells (Carrillo et al. 2015). Each R7 subtype promotes the survival of the class of Dm8 cells ( $y$ or pDm8) with which it synapses (Courgeon and Desplan 2019; Menon et al. 2019). The Dm8 dendrites that remain in the absence of R7 cells still project to the M6 layer (Courgeon and Desplan 2019), but it is not known whether R7 relies on Dm8 for targeting or survival information. Many synapses between R7 and Dm8 also include the projection neurons Tm5a (yR7) or Tm5b (pR7) as a second postsynaptic element (Gao et al. 2008; Takemura et al. 2015; Menon et al. 2019). In addition, Dm9 interneurons are both preand postsynaptic to R7 and mediate center-surround inhibition, similarly to horizontal cells in the mammalian retina (Takemura et al. 2013; Takemura et al. 2015; Heath et al. 2020). Our data indicate that the level of Loaf in Tm5a/b and Dm9 is more important for R7 targeting than 
its level in Dm8, suggesting that these cells may determine the stability of R7 contacts in the M6 layer. However, we cannot rule out the possibility that the drivers we used to express Loaf in Dm8 did not cause a phenotype because the level or timing of expression was not optimal.

\section{An excess of Loaf in the postsynaptic cells may destabilize R7 connections}

Our observation that the absence of Loaf from R7 only causes a phenotype when Loaf is present in its postsynaptic partners implies that Loaf is not essential for R7 targeting. In loaf mutants, redundant mechanisms must stabilize R7 terminals in the M6 layer; cell surface protein interactions often only specify a preference for one synaptic partner over another (Xu et al. 2019). Synaptic connections may not form entirely normally in these conditions, as loaf mutants show a reduced sensitivity to ultraviolet light when compared to isogenic controls (C.H. Lee, pers. comm.). Importantly, R7 mistargeting is much more striking when loaf is absent from photoreceptors, but present in the brain. We were able to reproduce this mistargeting by expressing loaf only in subsets of neurons in the brain that include the major postsynaptic partners of R7. The most parsimonious explanation for these phenotypes is that a mismatch in Loaf expression between R7 and its partners results in mistargeting. A similar phenomenon was observed for the homophilic cell adhesion molecule Klingon, which affects synapse formation when removed from either $\mathrm{R} 7$ or glial cells, but not when removed from both simultaneously (Shimozono et al. 2019). Matching pre- and postsynaptic levels are also important for the Drosophila Teneurin proteins to promote synapse formation (Hong et al. 2012; Mosca et al. 2012). This type of level matching, in which the presence of a protein in only one of the two partners is more deleterious than its absence from both, is well suited to refining synaptic specificity by eliminating inappropriate connections.

Interestingly, Loaf matching seems to be asymmetric; R7 mistargeting results if Loaf is absent in R7 and present in the postsynaptic cell, but not if it is absent in the postsynaptic cell and present in R7 (Fig. 5I). It is possible that matching levels in some way neutralize the activity of Loaf, or of a cell surface molecule regulated by Loaf. An excess of this molecule on the postsynaptic cell might prevent it from initiating or stabilizing synapses with $\mathrm{R} 7$, or drive it to preferentially connect with other neurons. However, the asymmetry could also reflect the 
presence of Loaf in multiple postsynaptic cells; loss of loaf from only one cell type may not be sufficient to disrupt R7 targeting.

\section{Loaf may control the trafficking of a cell surface molecule}

Our results suggest that Loaf does not itself act as a cell surface adhesion molecule. When epitope-tagged or untagged forms of Loaf are overexpressed in photoreceptors or cultured cells, they localize to intracellular vesicles that include endosomes and do not appear to reach the cell surface. In addition, they do not induce cell aggregation, further arguing against a homophilic adhesion function. CUB domains are present in a variety of functionally distinct proteins, and are thought to bind protein ligands, sometimes in combination with calcium ions (Gaboriaud et al. 2011). Some CUB domain proteins are involved in endocytosis of other molecules (Moestrup and Verroust 2001; Xu and Wang 2016), while members of the Neuropilin and Tolloid-like (Neto) family of CUB-LDL proteins are required for the normal localization and activity of glutamate receptors and other postsynaptic proteins (Zheng et al. 2004; Kim et al. 2012; Ramos et al. 2015; Sheng et al. 2015). It is thus possible that Loaf controls the level of other proteins on the cell surface by mediating their trafficking or endocytosis. The endosomal protein Commissureless functions in this manner, by trafficking the Roundabout axon guidance receptor directly from the Golgi to endosomes so that it does not reach the cell surface (Keleman et al. 2002). In another example, Rab6 and its activator Rich traffic Ncad to the cell surface, facilitating R7 targeting (Tong et al. 2011). Differences in Neurexin levels between axons and dendrites are also dependent on endocytosis and sorting (Ribeiro et al. 2019), and trafficking of synaptic adhesion molecules in general is highly regulated (Ribeiro et al. 2018).

Consistent with this model, we found that loss or gain of Loaf affects the function of coexpressed Lrp4, a presynaptic organizer in the olfactory system that has postsynaptic functions at mammalian neuromuscular junctions (Yumoto et al. 2012; Mosca et al. 2017). However, Lrp4 alone cannot explain the effects of Loaf, as removing loaf from photoreceptors still affects $\mathrm{R} 7$ targeting in an Lrp4 null mutant. Loaf may act through a protein similar to Lrp4, or through a combination of proteins. Alternatively, it is possible that under some conditions, 
perhaps in the presence of other interacting proteins, Loaf itself can reach the cell surface and function there. Some synaptic organizing molecules are transported to axons in lysosomerelated vesicles and secreted in a regulated manner (Arantes and Andrews 2006; Vukoja et al. 2018; Ibata et al. 2019). Further study of the mechanism of Loaf action will provide insight into the cellular mechanisms that enable synaptic connections to be stabilized only on the appropriate cells as neural circuits develop. 


\section{Materials and Methods}

\section{Fly stocks and genetics}

Fly stocks used were Rh5-GFP (Bloomington Drosophila Stock Center [BDSC] \#8600); Rh6-GFP (BDSC \#7461); gl-lacZ (Moses and Rubin 1991), R22E09-LexA, LexAop-myrTomato; GMR-GAL4 (Pecot et al. 2013); ey3.5-FLP, Act>CD2>GAL4 (BDSC \#35542 and \#4780); IGMRGAL4 (BDSC \#8605); UAS-loaf RNAiBL P\{TRiP.JF03040\}attP2 (BDSC \#28625); UAS-loaf RNAiKK P\{KK112220\}VIE-260B (Vienna Drosophila Resource Center [VDRC] \#102704); UAS-Lar RNAi P\{KK100581\}VIE-260B (VDRC \#107996); UAS-dcr2 (BDSC \#24650); panR7-lacZ (Hofmeyer et al. 2006); nos-Cas9 (BDSC \#54591); UAS-Cas9-P2 (BDSC \#58986); DIP- $\gamma$-GAL4 (Carrillo et al. 2015); tj-GAL4 ${ }^{N P 1624}$ (Kyoto Stock Center \#104055); drf-GAL4 (Brody et al. 2012); Mi\{PT-

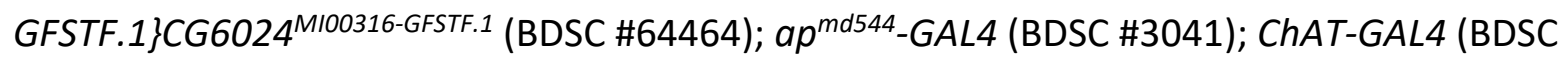
\#6798); repo-GAL4 (BDSC \#7415); hth-GAL4 (Wernet et al. 2003); bsh-GAL4 (Hasegawa et al. 2011); Vsx-GAL4 (BDSC \#29031); VGlut-GAL4 (BDSC \#26160); GMR9D03-GAL4 (BDSC \#40726); GH146-GAL4 (BDSC \#30026); dve NP3428-GAL4 (Kyoto Stock Center \#113273); vg-GAL4 (BDSC \#6819); ort ${ }^{\mathrm{C1a}}-$ GAL4 (BDSC \#56519); ort ${ }^{\mathrm{C2b}}$-GAL4 (Ting et al. 2014); GMR64H01-GAL4 (BDSC \#39322); UAS-LarHA (Hofmeyer and Treisman 2009); UAS-Lrp4HA; Lrp4 ${ }^{\text {dalek }}$ (Mosca et al. 2017); Liprin- $\alpha^{\text {oos }}$ (Hofmeyer et al. 2006); GMR9D03-DBD (BDSC \#68766); GMR38H04-AD (BDSC \#75758); MCFO-1 (Nern et al. 2015) and TSG101² (Moberg et al. 2005).

loaf mutant clones and loaf mutant clones overexpressing other proteins were generated using ey3.5-FLP, UAS-CD8GFP; IGMR-GAL4; FRT80, tub-GAL80. Ioaf MiMIC-GFSTF clones were generated using eyFLP; IGMR-GAL4, UAS-myr-tdTomato; FRT80, tub-GAL80. Clones in which specific cell types were labeled were generated by crossing ort ${ }^{\mathrm{C2b}}$-GAL4 (or other GAL4 lines); FRT80 (or FRT80, loaf ${ }^{\triangle 33}$ ) to $h s-F L P 122$, UAS-CD8GFP; FRT80, tub-GAL80. Overexpression clones were generated by crossing UAS-LoafHA (or UAS-Loaf, UAS-LarHA or UAS-Lrp4HA); FRT82 to ey3.5-FLP, UAS-CD8GFP; IGMR-GAL4; FRT82, tub-GAL80. To obtain sparse labelling of Tm5a/b/c neurons, flies with the genotype hsflp2PEST; UAS>stop>CD4-tdGFP/CyO; GMR9D03GAL4 were heat-shocked for $7 \mathrm{~min}$ at late L3 stage and dissected in the adult. loaf mutant clones in a background of Lrp4 overexpression were generated by crossing UAS-Lrp4HA; IGMR- 
GAL4, FRT80, loaf ${ }^{433}$ to eyFLP; FRT80, ubi-RFP. Lrp4 mutant clones were generated using ey-FLP, tub-GAL80, FRT19; IGMR-GAL4, UAS-CD8-GFP. To restore Loaf to specific cell types in a loaf mutant background, tj-GAL4 (or other GAL4 lines); loaf ${ }^{433} / S M 6-T M 6 B$ was crossed to UASLoafHA, UAS-myrTomato; loaf 433 /SM6-TM6B or to UAS-LoafHA; panR7-lacZ, loaf $433 / S M 6-T M 6 B$. GAL4 lines on the third chromosome were recombined with loaf ${ }^{\Delta 33}$ and recombinants carrying loaf were identified by PCR, except for GAL4 lines inserted at the attP2 site, which is very close to loaf. In these cases, new loaf alleles were directly introduced by CRISPR onto the GAL4 chromosome using nos-Cas9 and our transgenic loaf sgRNA flies, and identified by PCR.

\section{Immunohistochemistry}

Adult heads were dissected in cold 0.1 M sodium phosphate buffer (PB) pH 7.4, fixed in $4 \%$ formaldehyde in PB for $4 \mathrm{~h}$ at $4^{\circ} \mathrm{C}$ and washed in PB. Heads were then submerged in a sucrose gradient $(5 \%, 10 \%, 20 \%)$ and left in $25 \%$ sucrose overnight at $4^{\circ} \mathrm{C}$ for cryoprotection. Heads were embedded in OCT tissue freezing medium and frozen in dry ice/ethanol, and $12 \mu \mathrm{m}$ sections were cut on a cryostat. Sections were post-fixed in $0.5 \%$ formaldehyde in PB for $30 \mathrm{~min}$ at room temperature and washed three times in PB with $0.1 \%$ Triton (PBT) before incubation with primary antibodies overnight at $4^{\circ} \mathrm{C}$. Sections were washed 4 times for 20 min with PBT and incubated with secondary antibodies for $2 \mathrm{~h}$ at room temperature. Sections were washed again 4 times for 20 min before mounting in Fluoromount-G (Southern Biotech).

Pupal and adult whole brains were fixed in 4\% paraformaldehyde in PBS for $30 \mathrm{~min}$ at room temperature, washed 3 times for $10 \mathrm{~min}$ in PBST (PBS + 0.4\% Triton-X 100) and blocked in PBST $+10 \%$ donkey serum prior to incubation with primary antibodies overnight at $4^{\circ} \mathrm{C}$. For Loaf staining of pupal brains, this incubation was extended to 4 days. Samples were washed in PBST 3 times for at least 1 hour each and incubated with secondary antibodies for 2.5 hours at room temperature. Samples were washed 3 times for 20 min in PBST and once in PBS before mounting in SlowFade Gold AntiFade reagent (Life Technologies) on bridge slides. Pupal retinas were fixed in $4 \%$ paraformaldehyde in PBS for 30 min on ice, washed for 15 min in PBT and incubated with primary antibodies overnight at $4^{\circ} \mathrm{C}$. Retinas were washed 3 times for 5 min with PBT, incubated with secondary antibodies for $2 \mathrm{~h}$ at $4^{\circ} \mathrm{C}$ and washed again 3 times for 5 
min before mounting in $80 \%$ glycerol in PBS. Confocal images were collected with Leica SP8 and Zeiss LSM510 confocal microscopes.

The primary antibodies used were mouse anti-Chp (1:50; Developmental Studies Hybridoma Bank [DSHB] 24B10), chicken anti-GFP (1:400; Life Technologies), rat anti-HA (1:50; Roche 3F10), rabbit anti- $\beta$ galactosidase (1:100, Fisher), rat anti-Ncad (1:50; DSHB), rabbit antidsRed (1:500; Takara Bio), guinea pig anti-Loaf (1:400, Proteintech), mouse anti-Cnx99A (1:10, DSHB 6-2-1), mouse anti-Hrs (1:10, DSHB 27-4), mouse anti-Rab7 (1:10, DSHB), rabbit antiATP6V1B1 (Vha55; 1:200, Abgent), rabbit anti-Arl8 (1:200; DSHB), rat anti-Elav (1:100, DSHB), mouse anti-Dac (1:100; DSHB mAbdac1-1), mouse anti-Notch (1:10; DSHB C17.9C6), mouse anti-Arm (1:10; DSHB N2 7A1), sheep anti-GFP (1:200, Bio-Rad \#4745-1051), rabbit anti-RFP (1:500; MBL International \#PM005), rabbit anti-V5 (1:1000; Abcam ab9116), mouse anti-FLAG (1:500, Sigma F3165), mouse anti-Dac (1:40; DSHB mAbdac2-3), rabbit anti-Bsh (1:1800)(Ozel et al. 2021), and guinea pig anti-Runt (1:600; GenScript). Rhodamine-phalloidin (Invitrogen R415) was used at 1:20. The Loaf polyclonal antibody was made by Proteintech using the cytoplasmic domain (aa 292-378) as an antigen. Guinea pig anti-serum was affinity purified. Guinea pig antiRunt was made by GenScript using the full-length protein as an antigen. Secondary antibodies (Jackson Immunoresearch and Life Technologies) were coupled to the fluorochromes Cy3, AlexaFluor 488, or AlexaFluor 647.

\section{Quantifications}

To quantify the R7 targeting defect, fluorescent image stacks of $12-\mu \mathrm{m}$ adult head sections labeled for $g l-l a c Z, R h 3 / 4-l a c Z$, or anti-24B10 were gathered in 0.5- $\mu \mathrm{m}$ steps. Maximum intensity projections were obtained and termini projecting beyond the R8 layer were counted as "R7 correctly targeted" and those stopping in the R8 layer were counted as "R7 incorrectly targeted." Termini in the R8 layer were counted as total cartridge number per section. The percentage of mistargeting R7s was calculated for each section, except that when the phenotype was scored in photoreceptor clones, the percentage was calculated from all mistargeting R7 axons within clones from all sections. To quantify defects in UAS-LRP4 clones, GFP-labelled R7 termini that contacted each other in the M3 layer were counted as "M3 
clumping", while termini hyperfasciculating in the M6 layer were counted as "M6 clumping." Three termini contacting each other were counted as two instances of M3 or M6 clumping depending on in which layer the clumps resided. GFP-labelled R7 termini that extended past the M6 layer were counted as "overshooting." A terminus that extended past the M6 layer and turned to contact another clone was counted both as "M6 clumping" and "overshooting." The percentage of R7s belonging to each of these groups was calculated from all the R7 clones within each section.

To quantify cell aggregates in S2 cell culture experiments, fluorescent image stacks from fixed cells that had been labeled for GFP and HA were gathered in $0.5-\mu \mathrm{m}$ steps. Each image was examined for GFP positive (control) or GFP and HA positive (Sdk or Loaf) cells that contacted each other as aggregates. The number of cells in each aggregate was counted for each image. To measure intracellular colocalization, single confocal slices were processed with a median filter with neighborhood of 1 in ImageJ and each channel was linear contrast enhanced to spread values evenly from 0 to 255. A rectangular ROI was drawn around the central region of a single ommatidium and ImageJ was used to calculate Pearson's correlation coefficient on each region with pixel intensity above a threshold of 16 out of a range of 255 , to eliminate background.

\section{Western blotting}

To extract proteins, adult heads were dissected and frozen on dry ice, and then homogenized in Laemmli buffer (4\% SDS, 20\% glycerol, $120 \mathrm{mM}$ Tris-Cl pH 6.8, 0.02\% bromophenol blue, $10 \%$ beta-mercaptoethanol). Samples were heated at $95^{\circ} \mathrm{C}$ for $5 \mathrm{~min}$ and loaded onto a SDS-PAGE gel. Gels were run first at 80 volts for $20 \mathrm{~min}$, then 100 volts for the remainder of the time and transferred onto nitrocellulose membranes (Bio-Rad) for one hour at 100 volts. Membranes were washed for $5 \mathrm{~min}$ in TBST (20mM Tris (pH 7.6), $136 \mathrm{mM} \mathrm{NaCl}, 0.2 \%$ Tween-20), and blocked in 5\% low-fat milk in TBST solution for one hour. Membranes were incubated overnight with primary antibody in TBST with $5 \%$ milk at $4^{\circ} \mathrm{C}$, washed 3 times for 10 minutes in TBST and incubated in horseradish peroxidase-conjugated secondary antibodies (1:10,000; Jackson ImmunoResearch) at room temperature in TBST with 5\% milk for $2 \mathrm{~h}$. 
Membranes were washed 3 times for $10 \mathrm{~min}$ in TBST and once for $10 \mathrm{~min}$ in TBS. Enhanced chemiluminescence (Thermo SuperSignal WestPico) was used to develop the blots. Primary antibodies used were guinea pig anti-Loaf (1:1000, Proteintech) and mouse anti ß-tubulin (1:10,000; Sigma, T4026).

\section{Cloning and transgenic lines}

UAS-Loaf-FLAG-HA is clone UFO07678 (Drosophila Genomics Resource Center). UASLoaf was cloned by inserting an Nhe I/Xba I fragment of clone UFO07678 into the Xba I site of pUAST-attB. Both constructs were integrated into the VK1 PhiC31 site at position 59D3. The loaf sgRNA sequences GCTGGTGATTACGTCGGTGA (loaf gRNA 1) and TGCGGGACCATCCGGGTACC (loaf gRNA 2) identified on www.flyrnai.org/crispr2 were made with gene synthesis in pUC57 (GenScript) and cloned into pCFD4 (Port et al. 2014) by Gibson assembly. The construct was integrated into the attP40 site at $25 \mathrm{C} 6$. These flies were crossed to nos-Cas 9 flies to make germline mosaic flies. The progeny of these flies were crossed to balancer flies and screened by PCR using primers outside the expected deletion (CGCACGAACTTTGTGACACT and CTCAAGTCAATCGGTCCTTCC). In loaf ${ }^{\Delta 20}$, the deletion extends from TACGTCGGTGA in gRNA1 through TGCGGG in sgRNA2, creating a frameshift and a stop codon after 30 novel amino acids. loaf $f^{\triangle 33}$ has the final CGGTGA of sgRNA replaced by GATT, and then deletes through TGCGGG in sgRNA2, creating a stop codon immediately following Thr 208 at the end of the CUB domain. Injections and screening of transgenic flies were carried out by Genetivision. A V5-Loaf construct in which the V5 epitope tag (GKPIPNPLLGLDST) was inserted following H90, 4 residues after the predicted signal peptide cleavage site, was synthesized by GenScript and cloned into pUASTattB using the EcoRI and Xbal sites.

\section{S2 cell culture and aggregation assay}

S2 cells were grown in Schneider's Drosophila Medium (GIBCO Invitrogen) with 10\% heat inactivated fetal bovine serum and 50 units $/ \mathrm{ml}$ penicillin-50 g/ml streptomycin (GIBCO Invitrogen) at $25^{\circ} \mathrm{C}$. Cells were spun down and resuspended in PBS. Poly-L-lysine-treated slides 
were covered with 0.1-0.2 $\mathrm{ml}$ of the cell suspension. Cells were fixed for $10 \mathrm{~min}$ at room temperature with $4 \%$ paraformaldehyde, permeabilized for 15 min with $0.2 \%$ PBT, then blocked with $10 \%$ normal donkey serum. Slides were incubated with primary antibodies overnight at $4^{\circ} \mathrm{C}$ in a humid chamber, washed 4 times with PBS, and incubated with secondary antibody at room temperature for 1-2 hours. Samples were washed 3 times with PBS before mounting with Vectashield (Vector Labs). To stain cell surface proteins, cells were incubated with primary antibody in PBS for 2 hours at room temperature prior to fixation. Pictures were collected on a Leica SP8 confocal microscope.

For aggregation assays, S2 cells were pelleted 48 hours after transient transfection using Effectene Transfection Reagent (Qiagen) and washed in fresh medium. $2.5 \mathrm{ml}$ of cells at a concentration of $4 \times 10^{6}$ cells $/ \mathrm{ml}$ were rocked at $50 \mathrm{rpm}$ for at least 3 hours. Plates were then analyzed for the presence of cell aggregates. Pictures were collected on a Zeiss AxioZoom microscope. 


\section{Acknowledgements}

We thank Steve Cohen, Max Courgeon, Chi-Hon Lee, Ken Moberg, Tim Mosca, Larry Zipursky, the Bloomington Drosophila Stock Center, the Vienna Drosophila Resource Center, the Kyoto Stock Center, the Drosophila Genomics Resource Center and the Developmental Studies Hybridoma Bank for fly stocks and reagents, and Flybase for invaluable information. We thank Michael Cammer of the NYU Langone Microscopy Laboratory, which is supported by grant P30CA016087, for help with quantifying colocalization. We are grateful to Justine Oyallon for her contributions to the early stages of the project, and to Hui Hua Liu and DanQing He for technical assistance. The manuscript was improved by the critical comments of Hongsu Wang. This work was supported by NIH grants R01GM089799 and R01NS112211 to J.E.T. and by fellowship F31EY025568 to J.D. I.H. was supported by a Human Frontier Science Program postdoctoral fellowship (LT000757/2017).

Competing interests: The authors declare no competing interests. 


\section{References}

Akin O, Bajar BT, Keles MF, Frye MA, Zipursky SL. 2019. Cell-type-specific patterned stimulusindependent neuronal activity in the Drosophila visual system during synapse formation. Neuron 101: 894-904.

Arantes RM, Andrews NW. 2006. A role for synaptotagmin VII-regulated exocytosis of lysosomes in neurite outgrowth from primary sympathetic neurons. J Neurosci 26: 46304637.

Astigarraga S, Douthit J, Tarnogorska D, Creamer MS, Mano O, Clark DA, Meinertzhagen IA, Treisman JE. 2018. Drosophila Sidekick is required in developing photoreceptors to enable visual motion detection. Development 145: pii: dev158246. .

Bazigou E, Apitz H, Johansson J, Loren CE, Hirst EM, Chen PL, Palmer RH, Salecker I. 2007. Anterograde Jelly belly and Alk receptor tyrosine kinase signaling mediates retinal axon targeting in Drosophila. Cell 128: 961-975.

Bomkamp C, Padmanabhan N, Karimi B, Ge Y, Chao JT, Loewen CJR, Siddiqui TJ, Craig AM. 2019. Mechanisms of PTPsigma-mediated presynaptic differentiation. Front Synaptic Neurosci 11: 17.

Brody T, Yavatkar AS, Kuzin A, Kundu M, Tyson LJ, Ross J, Lin TY, Lee CH, Awasaki T, Lee T, Odenwald WF. 2012. Use of a Drosophila genome-wide conserved sequence database to identify functionally related cis-regulatory enhancers. Dev Dyn 241: 169-189.

Carrillo RA, Ozkan E, Menon KP, Nagarkar-Jaiswal S, Lee PT, Jeon M, Birnbaum ME, Bellen HJ, Garcia KC, Zinn K. 2015. Control of synaptic connectivity by a network of Drosophila IgSF cell surface proteins. Cell 163: 1770-1782.

Choe KM, Prakash S, Bright A, Clandinin TR. 2006. Liprin-alpha is required for photoreceptor target selection in Drosophila. Proc Natl Acad Sci U S A 103: 11601-11606.

Clandinin TR, Lee CH, Herman T, Lee RC, Yang AY, Ovasapyan S, Zipursky SL. 2001. Drosophila LAR regulates R1-R6 and R7 target specificity in the visual system. Neuron 32: 237-248.

Courgeon M, Desplan C. 2019. Coordination between stochastic and deterministic specification in the Drosophila visual system. Science 366: pii: eaay6727.

Culi J, Springer TA, Mann RS. 2004. Boca-dependent maturation of beta-propeller/EGF modules in low-density lipoprotein receptor proteins. EMBO J 23: 1372-1380.

Davis FP, Nern A, Picard S, Reiser MB, Rubin GM, Eddy SR, Henry GL. 2020. A genetic, genomic, and computational resource for exploring neural circuit function. Elife 9: pii: e50901.

Erclik T, Li X, Courgeon M, Bertet C, Chen Z, Baumert R, Ng J, Koo C, Arain U, Behnia R, Del Valle Rodriguez A, Senderowicz L, Negre N, White KP, Desplan C. 2017. Integration of temporal and spatial patterning generates neural diversity. Nature 541: 365-370.

Freeman M. 1996. Reiterative use of the EGF receptor triggers differentiation of all cell types in the Drosophila eye. Cell 87: 651-660.

Gaboriaud C, Gregory-Pauron L, Teillet F, Thielens NM, Bally I, Arlaud GJ. 2011. Structure and properties of the $\mathrm{Ca}^{2+}$-binding CUB domain, a widespread ligand-recognition unit involved in major biological functions. Biochem J 439: 185-193.

Gao S, Takemura SY, Ting CY, Huang S, Lu Z, Luan H, Rister J, Thum AS, Yang M, Hong ST, Wang JW, Odenwald WF, White BH, Meinertzhagen IA, Lee CH. 2008. The neural substrate of spectral preference in Drosophila. Neuron 60: 328-342. 
Gilbert J, Man HY. 2017. Fundamental elements in autism: from neurogenesis and neurite growth to synaptic plasticity. Front Cell Neurosci 11: 359.

Hakeda-Suzuki S, Takechi H, Kawamura H, Suzuki T. 2017. Two receptor tyrosine phosphatases dictate the depth of axonal stabilizing layer in the visual system. Elife 6: pii: e31812.

Han C, Jan LY, Jan YN. 2011. Enhancer-driven membrane markers for analysis of nonautonomous mechanisms reveal neuron-glia interactions in Drosophila. Proc Natl Acad Sci U S A 108: 9673-9678.

Han KA, Jeon S, Um JW, Ko J. 2016. Emergent synapse organizers: LAR-RPTPs and their companions. Int Rev Cell Mol Biol 324: 39-65.

Hasegawa E, Kitada Y, Kaido M, Takayama R, Awasaki T, Tabata T, Sato M. 2011. Concentric zones, cell migration and neuronal circuits in the Drosophila visual center. Development 138: 983-993.

Heath SL, Christenson MP, Oriol E, Saavedra-Weisenhaus M, Kohn JR, Behnia R. 2020. Circuit mechanisms underlying chromatic encoding in Drosophila photoreceptors. Curr Biol 30: 264-275.

Hofmeyer K, Maurel-Zaffran C, Sink H, Treisman JE. 2006. Liprin-alpha has LAR-independent functions in R7 photoreceptor axon targeting. Proc Natl Acad Sci U S A 103: 1159511600.

Hofmeyer K, Treisman JE. 2009. The receptor protein tyrosine phosphatase LAR promotes R7 photoreceptor axon targeting by a phosphatase-independent signaling mechanism. Proc Natl Acad Sci U S A 106: 19399-19404.

Holbrook S, Finley JK, Lyons EL, Herman TG. 2012. Loss of syd-1 from R7 neurons disrupts two distinct phases of presynaptic development. J Neurosci 32: 18101-18111.

Hong W, Mosca TJ, Luo L. 2012. Teneurins instruct synaptic partner matching in an olfactory map. Nature 484: 201-207.

Ibata K, Kono M, Narumi S, Motohashi J, Kakegawa W, Kohda K, Yuzaki M. 2019. Activitydependent secretion of synaptic organizer Cbln1 from lysosomes in granule cell sxons. Neuron 102: 1184-1198.

Karuppudurai T, Lin TY, Ting CY, Pursley R, Melnattur KV, Diao F, White BH, Macpherson L, Gallio M, Pohida T, Lee $\mathrm{CH}$. 2014. A hard-wired glutamatergic circuit pools and relays UV signals to mediate spectral preference in Drosophila. Neuron 81: 603-615.

Keleman K, Rajagopalan S, Cleppien D, Teis D, Paiha K, Huber LA, Technau GM, Dickson BJ. 2002. Comm sorts Robo to control axon guidance at the Drosophila midline. Cell 110: 415-427.

Kim YJ, Bao H, Bonanno L, Zhang B, Serpe M. 2012. Drosophila Neto is essential for clustering glutamate receptors at the neuromuscular junction. Genes Dev 26: 974-987.

Konstantinides N, Kapuralin K, Fadil C, Barboza L, Satija R, Desplan C. 2018. Phenotypic convergence: distinct transcription factors regulate common terminal features. Cell 174: 622-635.

Kulkarni A, Ertekin D, Lee CH, Hummel T. 2016. Birth order dependent growth cone segregation determines synaptic layer identity in the Drosophila visual system. Elife 5: e13715.

Kurmangaliyev YZ, Yoo J, Valdes-Aleman J, Sanfilippo P, Zipursky SL. 2020. Transcriptional programs of circuit assembly in the Drosophila visual system. Neuron 108: 1045-1057. 
Lee $\mathrm{CH}$, Herman T, Clandinin TR, Lee R, Zipursky SL. 2001. N-cadherin regulates target specificity in the Drosophila visual system. Neuron 30: 437-450.

Li X, Erclik T, Bertet C, Chen Z, Voutev R, Venkatesh S, Morante J, Celik A, Desplan C. 2013. Temporal patterning of Drosophila medulla neuroblasts controls neural fates. Nature 498: 456-462.

Maurel-Zaffran C, Suzuki T, Gahmon G, Treisman JE, Dickson BJ. 2001. Cell-autonomous and nonautonomous functions of LAR in R7 photoreceptor axon targeting. Neuron 32: 225235.

Meissner GW, Nern A, Singer RH, Wong AM, Malkesman O, Long X. 2019. Mapping neurotransmitter identity in the whole-mount Drosophila brain using multiplex highthroughput fluorescence in situ hybridization. Genetics 211: 473-482.

Menon KP, Kulkarni V, Takemura SY, Anaya M, Zinn K. 2019. Interactions between Dpr11 and DIP-gamma control selection of amacrine neurons in Drosophila color vision circuits. Elife 8: e48935.

Moberg KH, Schelble S, Burdick SK, Hariharan IK. 2005. Mutations in erupted, the Drosophila ortholog of mammalian tumor susceptibility gene 101, elicit non-cell-autonomous overgrowth. Dev Cell 9: 699-710.

Moestrup SK, Verroust PJ. 2001. Megalin- and cubilin-mediated endocytosis of protein-bound vitamins, lipids, and hormones in polarized epithelia. Annu Rev Nutr 21: 407-428.

Morante J, Erclik T, Desplan C. 2011. Cell migration in Drosophila optic lobe neurons is controlled by eyeless/Pax6. Development 138: 687-693.

Mosca TJ, Hong W, Dani VS, Favaloro V, Luo L. 2012. Trans-synaptic Teneurin signalling in neuromuscular synapse organization and target choice. Nature 484: 237-241.

Mosca TJ, Luginbuhl DJ, Wang IE, Luo L. 2017. Presynaptic LRP4 promotes synapse number and function of excitatory CNS neurons. Elife 6: e27347.

Moses K, Ellis MC, Rubin GM. 1989. The glass gene encodes a zinc-finger protein required by Drosophila photoreceptor cells. Nature 340: 531-536.

Moses K, Rubin GM. 1991. glass encodes a site-specific DNA-binding protein that is regulated in response to positional signals in the developing Drosophila eye. Genes Dev 5: 583-593.

Naval-Sanchez M, Potier D, Haagen L, Sanchez M, Munck S, Van de Sande B, Casares F, Christiaens V, Aerts S. 2013. Comparative motif discovery combined with comparative transcriptomics yields accurate targetome and enhancer predictions. Genome Res 23: 74-88.

Nern A, Pfeiffer BD, Rubin GM. 2015. Optimized tools for multicolor stochastic labeling reveal diverse stereotyped cell arrangements in the fly visual system. Proc Natl Acad Sci U S A 112: E2967-2976.

Newsome TP, Asling B, Dickson BJ. 2000. Analysis of Drosophila photoreceptor axon guidance in eye-specific mosaics. Development 127: 851-860.

Ozel MN, Kulkarni A, Hasan A, Brummer J, Moldenhauer M, Daumann IM, Wolfenberg H, Dercksen VJ, Kiral FR, Weiser M, Prohaska S, Von Kliest M, Hiesinger PR. 2019. Serial synapse formation through filopodial competition for synaptic seeding factors. Dev Cell 50: 447-461.

Ozel MN, Langen M, Hassan BA, Hiesinger PR. 2015. Filopodial dynamics and growth cone stabilization in Drosophila visual circuit development. Elife 4: e10721. 
Ozel MN, Simon F, Jafari S, Holguera I, Chen YC, Benhra N, El-Danaf RN, Kapuralin K, Malin JA, Konstantinides N, Desplan C. 2021. Neuronal diversity and convergence in a visual system developmental atlas. Nature 589: 88-95.

Pappu KS, Morey M, Nern A, Spitzweck B, Dickson BJ, Zipursky SL. 2011. Robo-3--mediated repulsive interactions guide 88 axons during Drosophila visual system development. Proc Natl Acad Sci U S A 108: 7571-7576.

Pecot MY, Tadros W, Nern A, Bader M, Chen Y, Zipursky SL. 2013. Multiple interactions control synaptic layer specificity in the Drosophila visual system. Neuron 77: 299-310.

Petrovic M, Hummel T. 2008. Temporal identity in axonal target layer recognition. Nature 456: 800-803.

Plazaola-Sasieta H, Fernandez-Pineda A, Zhu Q, Morey M. 2017. Untangling the wiring of the Drosophila visual system: developmental principles and molecular strategies. $J$ Neurogenet 31: 231-249.

Port F, Chen HM, Lee T, Bullock SL. 2014. Optimized CRISPR/Cas tools for efficient germline and somatic genome engineering in Drosophila. Proc Natl Acad Sci U S A 111: E2967-2976.

Ramos Cl, Igiesuorobo O, Wang Q, Serpe M. 2015. Neto-mediated intracellular interactions shape postsynaptic composition at the Drosophila neuromuscular junction. PLoS Genet 11: e1005191.

Ribeiro LF, Verpoort B, de Wit J. 2018. Trafficking mechanisms of synaptogenic cell adhesion molecules. Mol Cell Neurosci 91: 34-47.

Ribeiro LF, Verpoort B, Nys J, Vennekens KM, Wierda KD, de Wit J. 2019. SorCS1-mediated sorting in dendrites maintains neurexin axonal surface polarization required for synaptic function. PLoS Biol 17: e3000466.

Schwabe T, Borycz JA, Meinertzhagen IA, Clandinin TR. 2014. Differential adhesion determines the organization of synaptic fascicles in the Drosophila visual system. Curr Biol 24: 13041313.

Selleck SB, Steller H. 1991. The influence of retinal innervation on neurogenesis in the first optic ganglion of Drosophila. Neuron 6: 83-99.

Sheng N, Shi YS, Lomash RM, Roche KW, Nicoll RA. 2015. Neto auxiliary proteins control both the trafficking and biophysical properties of the kainate receptor GluK1. Elife 4: e11682.

Shimozono M, Osaka J, Kato Y, Araki T, Kawamura H, Takechi H, Hakeda-Suzuki S, Suzuki T. 2019. Cell surface molecule, Klingon, mediates the refinement of synaptic specificity in the Drosophila visual system. Genes Cells 24: 496-510.

Takahashi H, Craig AM. 2013. Protein tyrosine phosphatases PTPdelta, PTPsigma, and LAR: presynaptic hubs for synapse organization. Trends Neurosci 36: 522-534.

Takemura SY, Bharioke A, Lu Z, Nern A, Vitaladevuni S, Rivlin PK, Katz WT, Olbris DJ, Plaza SM, Winston P, Zhao T, Horne JA, Fetter RD, Takemura S, Blazek K, Chang LA, Ogundeyi O, Saunders MA, Shapiro V, Sigmund C, Rubin GM, Scheffer LK, Meinertzhagen IA, Chklovskii DB. 2013. A visual motion detection circuit suggested by Drosophila connectomics. Nature 500: 175-181.

Takemura SY, Xu CS, Lu Z, Rivlin PK, Parag T, Olbris DJ, Plaza S, Zhao T, Katz WT, Umayam L, Weaver C, Hess HF, Horne JA, Nunez-Iglesias J, Aniceto R, Chang LA, Lauchie S, Nasca A, Ogundeyi O, Sigmund C, Takemura S, Tran J, Langille C, Le Lacheur K, McLin S, Shinomiya A, Chklovskii DB, Meinertzhagen IA, Scheffer LK. 2015. Synaptic circuits and their 
variations within different columns in the visual system of Drosophila. Proc Natl Acad Sci U S A 112: 13711-13716.

Ting CY, McQueen PG, Pandya N, Lin TY, Yang M, Reddy OV, O'Connor MB, McAuliffe M, Lee CH. 2014. Photoreceptor-derived Activin promotes dendritic termination and restricts the receptive fields of first-order interneurons in Drosophila. Neuron 81: 830-846.

Ting CY, Yonekura S, Chung P, Hsu SN, Robertson HM, Chiba A, Lee CH. 2005. Drosophila Ncadherin functions in the first stage of the two-stage layer-selection process of R7 photoreceptor afferents. Development 132: 953-963.

Tong C, Ohyama T, Tien AC, Rajan A, Haueter CM, Bellen HJ. 2011. Rich regulates target specificity of photoreceptor cells and N-cadherin trafficking in the Drosophila visual system via Rab6. Neuron 71: 447-459.

Triplett JW, Feldheim DA. 2012. Eph and ephrin signaling in the formation of topographic maps. Semin Cell Dev Biol 23: 7-15.

Van Battum EY, Brignani S, Pasterkamp RJ. 2015. Axon guidance proteins in neurological disorders. Lancet Neurol 14: 532-546.

Vukoja A, Rey U, Petzoldt AG, Ott C, Vollweiter D, Quentin C, Puchkov D, Reynolds E, Lehmann M, Hohensee S, Rosa S, Lipowsky R, Sigrist SJ, Haucke V. 2018. Presynaptic biogenesis requires axonal transport of lysosome-related vesicles. Neuron 99: 1216-1232.

Wagner T, Dieckmann M, Jaeger S, Weggen S, Pietrzik CU. 2013. Stx5 is a novel interactor of VLDL-R to affect its intracellular trafficking and processing. Exp Cell Res 319: 1956-1972.

Wernet MF, Labhart T, Baumann F, Mazzoni EO, Pichaud F, Desplan C. 2003. Homothorax switches function of Drosophila photoreceptors from color to polarized light sensors. Cell 115: 267-279.

Xu C, Theisen E, Maloney R, Peng J, Santiago I, Yapp C, Werkhoven Z, Rumbaut E, Shum B, Tarnogorska D, Borycz J, Tan L, Courgeon M, Griffin T, Levin R, Meinertzhagen IA, De Bivort B, Drugowitsch J, Pecot MY. 2019. Control of synaptic specificity by establishing a relative preference for synaptic partners. Neuron 103: 865-877.

Xu Y, Wang T. 2016. CULD is required for rhodopsin and TRPL channel endocytic trafficking and survival of photoreceptor cells. J Cell Sci 129: 394-405.

Yogev S, Shen K. 2014. Cellular and molecular mechanisms of synaptic specificity. Annu Rev Cell Dev Biol 30: 417-437.

Yonekura S, Xu L, Ting CY, Lee CH. 2007. Adhesive but not signaling activity of Drosophila $\mathrm{N}$ cadherin is essential for target selection of photoreceptor afferents. Dev Biol 304: 759770.

Yumoto N, Kim N, Burden SJ. 2012. Lrp4 is a retrograde signal for presynaptic differentiation at neuromuscular synapses. Nature 489: 438-442.

Zhang KX, Tan L, Pellegrini M, Zipursky SL, McEwen JM. 2016. Rapid changes in the translatome during the conversion of growth cones to synaptic terminals. Cell Rep 14: 1258-1271.

Zheng Y, Mellem JE, Brockie PJ, Madsen DM, Maricq AV. 2004. SOL-1 is a CUB-domain protein required for GLR-1 glutamate receptor function in C. elegans. Nature 427: 451-457. 